Research Article; Received: March 20, 2021; Accepted: July 29, 2021

\title{
STUDY STRONG SHEFFER STROKE NON-ASSOCIATIVE MV-ALGEBRAS BY FUZZY FILTERS
}

\author{
Tahsin ONER $^{1}$, Tuğçe KATICAN ${ }^{2}$, and Arsham BORUMAND SAEID ${ }^{3}$ \\ ${ }^{1}$ Department of Mathematics, Ege University, İzmir, TURKEY \\ ${ }^{2}$ Department of Mathematics, İzmir University of Economics, İzmir, TURKEY \\ ${ }^{3}$ Department of Pure Mathematics, Shahid Bahonar University of Kerman, Kerman, IRAN
}

Abstract. In this paper, some types of fuzzy filters of a strong Sheffer stroke non-associative MV-algebra (for short, strong Sheffer stroke NMV-algebra) are introduced. By presenting new properties of filters, we define a prime filter in this algebraic structure. Then (prime) fuzzy filters of a strong Sheffer stroke NMV-algebra are determined and some features are proved. Finally, we built quotient strong Sheffer stroke NMV-algebra by a fuzzy filter.

\section{INTRODUCTION}

Sheffer operation was introduced by H. M. Sheffer as a single binary operation on a Boolean algebra restated all Boolean operations or formulas 16]. Since it has all diods on the chip forming processor in a computer, producing a single diod for this operation is simpler and cheaper than to produce different diods for other Boolean operations. Therefore, it is applied to algebraic structures such as Boolean algebras ( [9], [16]), ortholattices [3, orthoimplication algebras [1], Hilbert algebras 11, UP-algebras 14 and BL-algebras 13. In recent times, Chajda et al. introduced and studied non-associative MV-algebras (briefly, NMV-algebras) ( [4] [5], 6]) because associativity of the binary relation of a MV-algebra causes serious problems in expert systems in artificial intelligence ( [2], 6]). Also, Oner et al. analyzed filters and neutrosophic structures on strong Sheffer stroke NMValgebras $([10,15])$. On the other side, the notion of fuzzy logic was originally introduced by Lotfi Zadeh 18 and has been developing expeditiously. Since these

2020 Mathematics Subject Classification. 06F05, 03G25, 03G10.

Keywords. Sheffer operation, strong Sheffer stroke NMV-algebra, fuzzy filter.

■tahsin.oner@ege.edu.tr-Corresponding author; tugce.katican@izmirekonomi.edu.tr; arsham@uk.ac.ir

(D) 0000-0002-6514-4027; 0000-0003-1186-6750; 0000-0001-9495-6027. 
concepts have an important position in classic or nonclassic logical algebras, it leads to interesting results $([7],[8], 12],[17])$.

In this study, basic concepts and new properties of a strong Sheffer stroke NMValgebra are presented. Then a (prime) filter of strong Sheffer stroke NMV-algebras is defined and some features examined. It is shown that a filter of a strong sheffer stroke NMV-algebra is prime if and only if it is not contained by another filter of this algebraic structure. Indeed, it is proved that a filter of a strong Sheffer stroke NMV-algebra is prime if and only if the quotient structure defined by the filter is totally ordered strong Sheffer stroke NMV-algebra and its cardinality is less than or equals to 2. By describing a (prime) fuzzy filter of strong Sheffer stroke NMValgebras, related notions are stated. It is proved that $\alpha$ is a (prime) fuzzy filter of a strong Sheffer stroke NMV-algebra if and only if $\alpha_{a}=\{x \in A: a \leq \alpha(x)\}$ is empty or a (prime) filter of $A$, for all $a \in[0,1]$. Besides, it is shown that $F$ is a (prime) filter of a strong Sheffer stroke NMV-algebra if and only if a fuzzy subset $\alpha_{F}$ defined by $F$ is a (prime) fuzzy filter of this algebraic structure. It is demonstrated that a strong Sheffer stroke NMV-algebra is totally ordered if and only if every fuzzy filter is prime if and only if the filter $\{1\}$ is prime. Also, we prove that a fuzzy filter $\alpha$ of a strong Sheffer stroke NMV-algebra is prime if and only if $\alpha_{h}$ is a prime fuzzy filter of this algebra, for a surjective endomorphism $h$ on this algebra, and that $\alpha_{h}=\alpha$ if and only if $h\left(\alpha_{a}\right)=\alpha_{a}$, for an automorphism $h$ on this algebra and $a \in \operatorname{Im}(\alpha)$. Finally, a congruence relation on a strong Sheffer stroke NMV-algebra is defined by a fuzzy filter, and so, a quotient strong Sheffer stroke NMV-algebra is constructed by means of the congruence relation. In fact, a fuzzy filter $\alpha$ of a strong Sheffer stroke NMV-algebra is prime if and only if the quotient structure is a totally ordered strong Sheffer stroke NMV-algebra and its cardinality is less than or equals to 2. In addition, it is shown that $\alpha \circ h$ is a fuzzy filter of $A$ and the quotient structures defined by the fuzzy filters $\alpha \circ h$ and $\alpha$ are isomorphic, for strong Sheffer stroke NMV-algebras $A$ and $B$, an epimorphism $h$ between these algebras and a fuzzy filter $\alpha$ of $B$. Consequently, it is stated that the class of all fuzzy filters of a strong Sheffer stroke NMV-algebra forms a complete lattice since the interval $[0,1]$ is a complete lattice and has important properties.

\section{Preliminaries}

In this section, basic definitions and notions about strong Sheffer stroke NMValgebras are presented.

Definition 1. [3] Let $\mathcal{A}=(A, \mid)$ be a groupoid. The operation $\mid$ on $A$ is said to be a Sheffer stroke operation if it satisfies the following conditions:

(S1) $x|y=y| x$,

(S2) $(x \mid x) \mid(x \mid y)=x$,

$(S 3) x|((y \mid z) \mid(y \mid z))=((x \mid y) \mid(x \mid y))| z$,

(S4) $(x \mid((x \mid x) \mid(y \mid y))) \mid(x \mid((x \mid x) \mid(y \mid y)))=x$. 
Definition 2. [4] A strong Sheffer stroke $N M V$-algebra is an algebra $(A, \mid, 1)$ of type $(2,0)$ satisfying the identities for all $x, y, z \in A$ :

(n1) $x|y \approx y| x$,

(n2) $x \mid 0 \approx 1$

(n3) $(x \mid 1) \mid 1 \approx x$,

(n4) $((x \mid 1) \mid y)|y \approx((y \mid 1) \mid x)| x$,

$(n 5)(x \mid 1) \mid((x \mid y) \mid 1) \approx 1$,

(n6) $x|((((x \mid y) \mid y) \mid z) \mid z)| 1) \approx 1$

where 0 denotes the algebraic constant $1 \mid 1$.

Lemma 1. [10] Let $(A, \mid, 1)$ be a strong Sheffer Stroke NMV-algebra. Then the binary relation $\leq$ defined by

$$
x \leq y \text { if and only if } x \mid(y \mid 1) \approx 1
$$

is a partial order on $A$. Hence, $(A, \leq)$ is a poset with the least element 0 and the greatest element 1 .

Lemma 2. [10] In a strong Sheffer stroke $N M V$-algebra A, the following properties hold for all $x, y, z \in A$ :

(i) $x \mid(x \mid 1) \approx 1$,

(ii) $x \leq y \Leftrightarrow y|1 \leq x| 1$,

(iii) $y \leq x \mid(y \mid 1)$,

(iv) $y|1 \leq x| y$,

(v) $x \leq(x \mid y) \mid y$,

(vi) $x \leq(((x \mid y) \mid y) \mid z) \mid z$,

(vii) $((x \mid y) \mid y)|y \approx x| y$,

(viii) $x|1 \approx x| x$,

(ix) $x \mid(x \mid x) \approx 1$,

(x) $1 \mid(x \mid x) \approx x$,

(xi) $x \leq y \Rightarrow y|z \leq x| z$,

(xii) $x|(y \mid 1) \leq(y \mid(z \mid 1))|((x \mid(z \mid 1)) \mid 1)$,

(xiii) $x|(y \mid 1) \leq(z \mid(x \mid 1))|((z \mid(y \mid 1)) \mid 1)$.

Definition 3. $[10]$ A nonempty subset $F \subseteq A$ is called a filter of $A$ if it satisfies the following properties:

$\left(S_{f}-1\right) 1 \in F$,

$\left(S_{f}-2\right)$ For all $x, y \in A, x \mid(y \mid 1) \in F$ and $x \in F$ imply $y \in F$.

Definition 4. [10] Let $F$ be a filter of a strong Sheffer stroke $N M V$-algebra $(A, \mid, 1)$.

Define the binary relation $\propto_{F}$ on $A$ as below: for all $x, y \in A$

$$
x \propto_{F} y \text { if and only if } x \mid(y \mid 1) \in F \text { and } y \mid(x \mid 1) \in F .
$$

Definition 5. [10] If $x \xi y$ implies $x|k \xi y| k$, for all $x, y, k \in A$, then the equivalence relation $\xi$ is called a congruence relation on $A$. 
Lemma 3. [10] An equivalence relation $\xi$ is a congruence relation on $A$ if and only if $x \xi y$ and $k_{1} \xi k_{2}$ imply $x\left|k_{1} \xi y\right| k_{2}$.

Lemma 4. [10] Let $F$ be a filter of a strong Sheffer stroke $N M V$-algebra $(A, \mid, 1)$ and the binary relation $\propto_{F}$ be defined as (1). Then $\propto_{F}$ is a congruence relation on $A$.

Theorem 1. [10] Let $F$ be a filter of a strong Sheffer stroke NMV-algebra $(A, \mid, 1)$ and $\propto$ be a congruence relation on $A$ defined by $F$. Then $\left(A / \propto,\left.\right|_{\propto},[1]_{\propto}\right)$ is also a strong Sheffer stroke NMV-algebra where $A / F \equiv A / \propto=\left\{[x]_{\propto}: x \in A\right\}$, the strong Sheffer stroke $\left.\right|_{\propto}$ on $A / F$ is defined by $\left.[x]_{\propto}\right|_{\propto}[y]_{\propto} \approx[x \mid y]_{\propto}$, for all $x, y \in A$ and $F \approx[1]_{\propto}$.

Definition 6. [10] Let $\left(A,\left.\right|_{A}, 1_{A}\right)$ and $\left(B,\left.\right|_{B}, 1_{B}\right)$ be strong Sheffer stroke $N M V$ algebras. A mapping $h: A \longrightarrow B$ is called a homomorphism if

$$
h\left(\left.x\right|_{A} y\right)=\left.h(x)\right|_{B} h(y),
$$

for all $x, y \in A$.

\section{Some Results in Strong Sheffer Stroke NMV-Algebras}

In this section, new properties of strong Sheffer stroke NMV-algebras are given. Unless otherwise stated, $A$ represents a strong Sheffer stroke NMV-algebra.

Lemma 5. Let $A$ be a strong Sheffer stroke $N M V$-algebra. Then $(A, \leq)$ is a bounded lattice with the least element 0 and the greatest element 1 of $A$, where $x \vee y \approx$ $(x \mid(y \mid 1)) \mid(y \mid 1)$ and $x \wedge y \approx(((x \mid 1) \mid y) \mid y) \mid 1$, for all $x, y \in A$.

Proof. It is known from Lemma 1 that $(A, \leq)$ is a poset. Then $x \leq(x \mid(y \mid 1)) \mid(y \mid 1)$ and $y \leq(x \mid(y \mid 1)) \mid(y \mid 1)$ from Lemma 2 (v) and (iii), respectively. Thus, $(x \mid(y \mid 1)) \mid(y \mid$ 1 ) is an upper bound of $x$ and $y$. Let $x, y \leq z$. So, $x \mid(z \mid 1) \approx 1$ and $y \mid(z \mid 1) \approx 1$ from Lemma 1. Since

$$
\begin{aligned}
(x \mid(y \mid 1)) \mid(y \mid 1) & \leq(z \mid(y \mid 1)) \mid(y \mid 1) \\
& \approx(((z \mid 1) \mid 1) \mid(y \mid 1)) \mid(y \mid 1) \\
& \approx(((y \mid 1) \mid 1) \mid(z \mid 1)) \mid(z \mid 1) \\
& \approx(y \mid(z \mid 1)) \mid(z \mid 1) \\
& \approx(z \mid 1) \mid 1 \\
& \approx z
\end{aligned}
$$

from Lemma 2 (i), (xi), (n1), (n3) and (n4), it follows that $(x \mid(y \mid 1)) \mid(y \mid 1)$ is the least upper bound of $x$ and $y$. Hence, $x \vee y \approx(x \mid(y \mid 1)) \mid(y \mid 1)$, and similarly, $x \wedge y \approx$ $(((x \mid 1) \mid y) \mid y) \mid 1$, for all $x, y \in A$.

Since $0|(x \mid 1) \approx(x \mid 1)| 0 \approx 1$ and $x|(1 \mid 1) \approx x| 0 \approx 1$ from (n1) and (n2), it is obtained from Lemma 1 that $0 \leq x$ and $x \leq 1$, for all $x, y \in A$. Therefore, 0 is the least element and 1 is the greatest element of $A$ 
Proposition 1. Let $A$ be a strong Sheffer stroke $N M V$-algebra. Then

$$
x|((y \mid(z \mid 1)) \mid 1) \approx(x \mid(y \mid 1))|((x \mid(z \mid 1)) \mid 1),
$$

for all $x, y, z \in A$.

Proof. Let $A$ be a strong Sheffer stroke NMV-algebra.

$$
\begin{aligned}
x \mid((y \mid(z \mid 1)) \mid 1) & \approx x \mid((y \mid(z \mid 1)) \mid(y \mid(z \mid 1))) \\
& \approx y \mid((x \mid(z \mid 1)) \mid(x \mid(z \mid 1))) \\
& \approx y \mid((x \mid(z \mid 1)) \mid 1) \\
& \geq(x \mid(y \mid 1)) \mid((x \mid(z \mid 1)) \mid 1)
\end{aligned}
$$

from Lemma 2 (viii), (iii), (xi), (S1) and (S3). Also,

$$
\begin{aligned}
x \mid((y \mid(z \mid 1)) \mid 1) & \approx x \mid((y \mid(z \mid 1)) \mid(y \mid(z \mid 1))) \\
& \approx y \mid((x \mid(z \mid 1)) \mid(x \mid(z \mid 1))) \\
& \approx y \mid((x \mid(z \mid 1)) \mid 1) \\
& \leq(x \mid(y \mid 1)) \mid((x \mid((x \mid(z \mid 1)) \mid 1)) \mid 1) \\
& \approx(x \mid(y \mid 1)) \mid((x \mid((x \mid(z \mid 1)) \mid(x \mid(z \mid 1)))) \mid 1) \\
& \approx(x \mid(y \mid 1))|(((x \mid x) \mid(x \mid x)) \mid(z \mid 1))| 1) \\
& \approx(x \mid(y \mid 1)) \mid((x \mid(z \mid 1)) \mid 1)
\end{aligned}
$$

from Lemma 2 (viii), (xiii), (S1)-(S3).

Hence, $x|((y \mid(z \mid 1)) \mid 1) \approx(x \mid(y \mid 1))|((x \mid(z \mid 1)) \mid 1)$, for all $x, y, z \in A$.

Proposition 2. Let $A$ be a strong Sheffer stroke NMV-algebra. Then

$$
(x \mid y) \mid 1 \leq x \text { and }(x \mid y) \mid 1 \leq y,
$$

for all $x, y \in A$.

Proof. Let $A$ be a strong Sheffer stroke NMV-algebra. Since $((x \mid y) \mid 1) \mid(x \mid 1) \approx$ $(x \mid 1) \mid((x \mid y) \mid 1) \approx 1$ and $((x \mid y) \mid 1)|(y \mid 1) \approx(y \mid 1)|((y \mid x) \mid 1) \approx 1$ from $(\mathrm{n} 1)$ and $(\mathrm{n} 5)$, it is obtained from Lemma 1 that $(x \mid y) \mid 1 \leq x$ and $(x \mid y) \mid 1 \leq y$, for all $x, y \in A$.

Lemma 6. A nonempty subset $F$ of $A$ is a filter of $A$ if and only if $\left(S_{f}-3\right) x, y \in F$ imply $(x \mid y) \mid 1 \in F$,

$\left(S_{f}-4\right) x \in F$ and $x \leq y$ imply $y \in F$, for all $x, y \in A$.

Proof. $(\Rightarrow)$ Let $F$ be a filter of $A$ and $x, y \in A$. Since

$$
\begin{aligned}
x \mid(((x \mid y) \mid y) \mid 1) & \approx x \mid(((x \mid y) \mid y) \mid((x \mid y) \mid y)) \\
& \approx(x \mid y) \mid((x \mid y) \mid(x \mid y)) \\
& \approx 1
\end{aligned}
$$

from Lemma 2 (viii), (ix), (S1) and (S3), it follows from $\left(S_{f}-2\right)$ that $(x \mid y) \mid y \in F$. Since $y|(((x \mid y) \mid 1) \mid 1)=(x \mid y)| y \in F$ from (n1) and (n3), respectively, it is obtained 
from $\left(S_{f}-2\right)$ that $(x \mid y) \mid 1 \in F$. Let $x \in F$ and $x \leq y$. Then $x \mid(y \mid 1) \in F$ from Lemma 1 and $\left(S_{f}-1\right)$. Thus, $y \in F$ from $\left(S_{f}-2\right)$.

$(\Leftarrow)$ Let $F$ be a nonempty subset of $A$ satisfying $\left(S_{f}-3\right)$ and $\left(S_{f}-4\right)$. Assume that $x \in F$. Since $x \leq 1$ for all $x \in A$, it follows from $\left(S_{f}-4\right)$ that $1 \in F$. Let $x \mid(y \mid 1) \in F$ and $x \in F$. Then $(x \mid(x \mid(y \mid 1))) \mid 1 \in F$ from $\left(S_{f}-3\right)$. Since

$$
\begin{aligned}
((x \mid(x \mid(y \mid 1))) \mid 1) \mid(y \mid 1) & \approx((((y \mid 1) \mid x) \mid x) \mid 1) \mid(y \mid 1) \\
& \approx((((x \mid 1) \mid y) \mid y) \mid 1) \mid(y \mid 1) \\
& \approx(y \mid 1) \mid((y \mid(y \mid(x \mid 1))) \mid 1) \\
& \approx 1
\end{aligned}
$$

from (n1), (n4) and (n5), it is obtained from Lemma 1 that $(x \mid(x \mid(y \mid 1))) \mid 1 \leq y$. Thus, $y \in F$ from $\left(S_{f}-4\right)$.

Lemma 7. Let $F$ be a filter of $A$. Then

(a) $z \mid(((y \mid(x \mid 1)) \mid(x \mid 1)) \mid 1) \in F$ and $z \in F$ imply $(x \mid(y \mid 1)) \mid(y \mid 1) \in F$,

(b) $z \mid((y \mid(x \mid 1)) \mid 1) \in F$ and $z \in F$ imply $((x \mid(y \mid 1)) \mid(y \mid 1)) \mid(x \mid 1) \in F$ and

(c) $x \mid((y \mid(z \mid 1)) \mid 1) \in F$ and $x \mid(y \mid 1) \in F$ imply $x \mid(z \mid 1) \in F$,

for all $x, y, z \in A$.

Proof. (a) Since

$$
\begin{aligned}
z \mid(((x \mid(y \mid 1)) \mid(y \mid 1)) \mid 1) & \approx z \mid(((((x \mid 1) \mid 1) \mid(y \mid 1)) \mid(y \mid 1)) \mid 1) \\
& \approx z \mid(((((y \mid 1) \mid 1) \mid(x \mid 1)) \mid(x \mid 1)) \mid 1) \\
& \approx z \mid(((y \mid(x \mid 1)) \mid(x \mid 1)) \mid 1) \in F
\end{aligned}
$$

from (n3) and (n4) and $z \in F$, it follows from $\left(S_{f}-2\right)$ that $(x \mid(y \mid 1)) \mid(y \mid 1) \in F$.

(b) Since

$$
\begin{aligned}
z \mid((((x \mid(y \mid 1)) \mid(y \mid 1)) \mid(x \mid 1)) \mid 1) & \approx z \mid((((((x \mid 1) \mid 1) \mid(y \mid 1)) \mid(y \mid 1)) \mid(x \mid 1)) \mid 1) \\
& \approx z \mid((((((y \mid 1) \mid 1) \mid(x \mid 1)) \mid(x \mid 1)) \mid(x \mid 1)) \mid 1) \\
& \approx z \mid((((y \mid(x \mid 1)) \mid(x \mid 1)) \mid(x \mid 1)) \mid 1) \\
& \approx z \mid((y \mid(x \mid 1)) \mid 1) \in F
\end{aligned}
$$

from (n3), (n4) and Lemma 2 (vii) and $z \in F$, it is obtained from $\left(S_{f}-2\right)$ that $((x \mid(y \mid 1)) \mid(y \mid 1)) \mid(x \mid 1) \in F$.

(c) Since $(x \mid(y \mid 1))|((x \mid(z \mid 1)) \mid 1) \approx x|((y \mid(z \mid 1)) \mid 1) \in F$ from Proposition 1 and $x \mid(y \mid 1) \in F$, it follows from $\left(S_{f}-2\right)$ that $x \mid(z \mid 1) \in F$.

Definition 7. Let $F$ be a filter of $A$. Then $F$ is a prime filter of $A$ if $x \vee y \in F$ implies $x \in F$ or $y \in F$, for all $x, y \in A$.

Example 1. Consider a strong Shefeer stroke $N M V$-algebra $(A, \mid, 1)$ where a set $A=\{0, a, b, c, d, e, f, 1\}$ and the operation $\mid$ on $A$ has the following Cayley table $([10]):$ 
TABle 1. Cayley table of

\begin{tabular}{c|llllllll}
$\mid$ & 0 & $a$ & $b$ & $c$ & $d$ & $e$ & $f$ & 1 \\
\hline 0 & 1 & 1 & 1 & 1 & 1 & 1 & 1 & 1 \\
$a$ & 1 & $f$ & 1 & 1 & $f$ & $f$ & 1 & $f$ \\
$b$ & 1 & 1 & $e$ & 1 & $e$ & 1 & $e$ & $e$ \\
$c$ & 1 & 1 & 1 & $d$ & 1 & $d$ & $d$ & $d$ \\
$d$ & 1 & $f$ & $e$ & 1 & $c$ & $f$ & $e$ & $c$ \\
$e$ & 1 & $f$ & 1 & $d$ & $f$ & $b$ & $d$ & $b$ \\
$f$ & 1 & 1 & $e$ & $d$ & $e$ & $d$ & $a$ & $a$ \\
1 & 1 & $f$ & $e$ & $d$ & $c$ & $b$ & $a$ & 0
\end{tabular}

Then $\{a, d, e, 1\}$ is a prime filter of $A$ while $\{e, 1\}$ is not since $a \notin\{e, 1\}$ and $c \notin\{e, 1\}$ when $a \vee c \approx(a \mid(c \mid 1))|(c \mid 1) \approx(a \mid d)| d \approx f \mid d \approx e \in\{e, 1\}$.

Lemma 8. Let $F$ be a filter of $A$. Then $F$ is a prime filter of $A$ if and only if $x \in F$ or $x \mid 1 \in F$, for all $x \in A$.

Proof. Let $F$ be a prime filter of $A$. Since

$$
\begin{aligned}
x \vee(x \mid 1) & \approx(x \mid((x \mid 1) \mid 1)) \mid((x \mid 1) \mid 1) \\
& \approx x \mid(x \mid x) \\
& \approx 1 \in F
\end{aligned}
$$

from Lemma 5. (n1), (n3), Lemma 2 (ix) and $\left(S_{f}-1\right)$, it is obtained that $x \in F$ or $x \mid 1 \in F$, for all $x \in A$.

Conversely, let $F$ be a filter of $A$ such that $x \in F$ or $x \mid 1 \in F$, for all $x \in A$. Assume that $x \vee y \in F$ such that $x \notin F$ and $y \notin F$, for some $x, y \in A$. Then $x \mid 1 \in F$ and $y \mid 1 \in F$. Since $x|1 \leq(y \mid 1)|((x \mid 1) \mid 1) \approx x \mid(y \mid 1)$ and $y|1 \leq(x \mid 1)|((y \mid 1) \mid 1) \approx$ $y \mid(x \mid 1)$ from Lemma 2 (iii), (n1) and (n3), it follows from $\left(S_{f}-4\right)$ that $x \mid(y \mid 1) \in F$ and $y \mid(x \mid 1) \in F$. Since $(x \mid(y \mid 1)) \mid(y \mid 1) \approx x \vee y \in F$ and $(y \mid(x \mid 1)) \mid(x \mid 1) \approx y \vee x \approx$ $x \vee y \in F$ from Lemma 5 , it is obtained from $\left(S_{f}-2\right)$ that $x \in F$ and $y \in F$. This is a contradiction. Thus, $x \vee y \in F$ implies $x \in F$ or $y \in F$ which means that $F$ is a prime filter of $A$.

Lemma 9. Let $F$ be a filter of $A$. Then $F$ is a prime filter of $A$ if and only if $x \notin F$ and $y \notin F$ imply $x \mid(y \mid 1) \in F$ and $y \mid(x \mid 1) \in F$, for all $x, y \in A$.

Proof. Let $F$ be a prime filter of $A, x \notin F$ and $y \notin F$. Then $x \mid 1 \in F$ and $y \mid 1 \in F$. Since $x|1 \leq(y \mid 1)|((x \mid 1) \mid 1) \approx x \mid(y \mid 1)$ and $y|1 \leq(x \mid 1)|((y \mid 1) \mid 1) \approx y \mid(x \mid 1)$ from Lemma 2 (iii), (n1) and (n3), it follows from $\left(S_{f}-4\right)$ that $x \mid(y \mid 1) \in F$ and $y \mid(x \mid 1) \in F$.

Conversely, let $F$ be a filter of $A$ such that $x \notin F$ and $y \notin F$ imply $x \mid(y \mid 1) \in F$ and $y \mid(x \mid 1) \in F$, for all $x, y \in A$. Assume that $x \notin F$ and $x \mid 1 \notin F$, for some $x \in A$. Then $x|1 \approx x| x \approx x \mid((x \mid 1) \mid 1) \in F$ and $x \approx(x \mid x)|(x \mid x) \approx(1 \mid((x \mid x) \mid(x \mid x)))|(1 \mid((x \mid x)$ 
$\mid(x \mid x))) \approx(x \mid 1) \mid(x \mid 1) \in F$ from (n3), Lemma 2 (viii), (x) and (S1)-(S2). This is a contradiction. Thus, $x \in F$ or $x \mid 1 \in F$, for all $x \in F$, i.e., $F$ is a prime filter of $A$.

Lemma 10. Let $F$ be a filter of $A$. Then

(i) $x \in F$ and $y \in F$ imply $x \wedge y \in F$,

(ii) $F$ is a prime filter of $A$ if and only if $x \mid(y \mid 1) \in F$ or $y \mid(x \mid 1) \in F$, for all $x, y \in A$.

Proof. $\quad$ (i) It is clear.

(ii) Let $F$ be a prime filter of $A$. Since

$$
\begin{aligned}
(x \mid(y \mid 1)) \vee(y \mid(x \mid 1)) & \approx((x \mid(y \mid 1)) \mid((y \mid(x \mid 1)) \mid 1)) \mid((y \mid(x \mid 1)) \mid 1) \\
& \approx((x \mid(y \mid 1)) \mid((y \mid(x \mid x)) \mid(y \mid(x \mid x)))) \mid((y \mid(x \mid x)) \mid 1) \\
& \approx((((x \mid(y \mid 1)) \mid(x \mid x)) \mid((x \mid(y \mid 1)) \mid(x \mid x))) \mid y) \mid((y \mid(x \mid x)) \mid 1) \\
& \approx(y \mid(x \mid x)) \mid((y \mid(x \mid x)) \mid 1) \\
& \approx 1 \in F
\end{aligned}
$$

from Lemma 5 Lemma2(i) and (viii), (S1)-(S3), it follows that $x \mid(y \mid 1) \in F$ or $y \mid(x \mid 1) \in F$

Conversely, let $F$ be a filter of $A$ such that $x \mid(y \mid 1) \in F$ or $y \mid(x \mid 1) \in F$, for all $x, y \in A$. Suppose that $x \vee y \in F$. If $x \mid(y \mid 1) \in F$, then we have from $\left(S_{f}-2\right)$ that $y \in F$ since $(x \mid(y \mid 1)) \mid(y \mid 1) \approx x \vee y \in F$ from Lemma 5 Similarly, if $y \mid(x \mid 1) \in F$, then we get from $\left(S_{f}-2\right)$ that $x \in F$ since $(y \mid(x \mid 1)) \mid(x \mid 1) \approx y \vee x \approx x \vee y \in F$ from Lemma 5. Hence, $F$ is a prime filter of $A$.

Corollary 1. Let $F$ be a filter of $A$ such that $F \neq A$. Then $F$ is a prime filter of $A$ if and only if $(x \mid(y \mid 1)) \vee(y \mid(x \mid 1)) \in F$, for all $x, y \in A$.

Lemma 11. Let $F$ be a filter of $A$ such that $F \neq A$. Then $F$ is a prime filter of $A$ if and only if there is no a filter $G$ of $A$ such that $F \subset G \subset A$.

Proof. Let $F$ be a prime filter of $A$. Assume that $G$ is a filter of $A$ such that $F \subset G \subset A$ and $y \in G$ such that $y \notin F$. Then $y \mid 1 \in F$, and so, $y \mid 1 \in G$. Since $y \in G$ and $y \mid 1 \in G$, it follows from Lemma 2 (ix), (n1), Lemma 5 and Lemma 10 (i) that

$$
\begin{aligned}
0 & \approx 1 \mid 1 \\
& \approx((y \mid 1) \mid((y \mid 1) \mid(y \mid 1))) \mid 1 \\
& \approx(((y \mid 1) \mid(y \mid 1)) \mid(y \mid 1)) \mid 1 \\
& \approx y \wedge(y \mid 1) \in G .
\end{aligned}
$$


Since $0 \in G$ and 0 is the least element of $A$, we have from $\left(S_{f}-4\right)$ that $x \in G$, for all $x \in A$. Thus, $G=A$ which is a contradiction. Therefore, there is no a filter $G$ of $A$ such that $F \subset G \subset A$.

Conversely, let there be no a filter $G$ of $A$ such that $F \subset G \subset A$. Suppose that $x \vee y \in F$ such that $x, y \notin F$. Then there exists a filter $G$ of $A$ such that $x \in G$ or $y \in G$. Since $x, y \leq x \vee y$, we have from $\left(S_{f}-4\right)$ that $x \vee y \in G$. Thus, $F \subset G$ which is a contradiction. Hence, $x \vee y \in F$ implies $x \in F$ or $y \in F$ which means that $F$ is a prime filter of $A$.

Lemma 12. Let $F$ be a filter of $A$ and $\propto_{F}$ be a congruence relation on $A$ defined by $F$. Define a relation $\subseteq$ on $A / F$ by

$$
[x]_{\propto_{F}} \subseteq[y]_{\propto_{F}} \Leftrightarrow x \mid(y \mid 1) \in F,
$$

for all $x, y \in A$. Then the relation $\subseteq$ is a partial order on $A / F$.

Proof. Let $F$ be a filter of $A$ and $\propto_{F}$ be a congruence relation on $A$ defined by $F$. Then $\left(A / F,\left.\right|_{\alpha_{F}}, F\right)$ is a strong Sheffer stroke NMV-algebra by Theorem 1

- Since $x \mid(x \mid 1) \approx 1 \in F$ from Lemma 2 (i) and $\left(S_{f}-1\right)$, it follows that $[x]_{\propto_{F}} \subseteq$ $[x]_{\propto_{F}}$, for all $x \in A$.

- Let $[x]_{\propto_{F}} \subseteq[y]_{\propto_{F}}$ and $[y]_{\propto_{F}} \subseteq[x]_{\propto_{F}}$. Then $x \mid(y \mid 1) \in F$ and $y \mid(x \mid 1) \in F$, and so, $x \propto_{F} y$. Thus, $[x]_{\propto_{F}}=[y]_{\propto_{F}}$.

- Let $[x]_{\propto_{F}} \subseteq[y]_{\propto_{F}}$ and $[y]_{\propto_{F}} \subseteq[z]_{\propto_{F}}$. Then $x \mid(y \mid 1) \in F$ and $y \mid(z \mid 1) \in F$. Since $x|(y \mid 1) \leq(y \mid(z \mid 1))|((x \mid(z \mid 1)) \mid 1)$ from Lemma 2 (xii), it is obtained from $\left(S_{f}-4\right)$ that $(y \mid(z \mid 1)) \mid((x \mid(z \mid 1)) \mid 1) \in F$. Thus, it follows from $\left(S_{f}-2\right)$ that $x \mid(z \mid 1) \in F$ which implies that $[x]_{\propto_{F}} \subseteq[z]_{\propto_{F}}$.

Hence, the relation $\subseteq$ is a partial order on $A / F$.

Theorem 2. Let $F$ be a filter of $A$ and $\propto_{F}$ be a congruence relation on $A$ defined by $F$. Then $F$ is a prime filter of $A$ if and only if $\left(A / F,\left.\right|_{\alpha_{F}}, F\right)$ is totally ordered and $|A / F| \leq 2$.

Proof. Let $F$ be a filter of $A$ and $\propto_{F}$ be a congruence relation on $A$ defined by $F$. Then $\left(A / F,\left.\right|_{\alpha_{F}}, F\right)$ is a strong Sheffer stroke NMV-algebra by Theorem 1. Let $F$ be a prime filter of $A$. Then $x \mid(y \mid 1) \in F$ or $y \mid(x \mid 1) \in F$ by Lemma 10 (ii). Thus, $[x]_{\propto_{F}} \subseteq[y]_{\propto_{F}}$ or $[y]_{\propto_{F}} \subseteq[x]_{\propto_{F}}$ from Lemma 12 . Hence, $\left(A / F,\left.\right|_{\alpha_{F}}, F\right)$ is totally ordered. Moreover, let $|A / F|>2$. Then $[x]_{\propto_{F}} \in A / F$ such that $[0]_{\propto_{F}} \subset[x]_{\propto_{F}} \subset$ $[1]_{\propto_{F}}$. Since $F$ is a prime filter of $A$, it is known that $x \in F$ or $x \mid 1 \in F$. Assume that $x \mid 1 \in F$. Since $x|(0 \mid 1) \approx x| 1 \in F$ and $0 \mid(x \mid 1) \approx 1 \in F$ from (n2), we get $[x]_{\propto_{F}}=[0]_{\propto_{F}}$ which is a contradiction. Therefore, $|A / F| \leq 2$.

Conversely, let $\left(A / F,\left.\right|_{\propto_{F}}, F\right)$ be totally ordered. Then $[x]_{\propto_{F}} \subseteq[y]_{\propto_{F}}$ or $[y]_{\propto_{F}} \subseteq$ $[x]_{\propto_{F}}$, for all $x, y \in A$. So, $x \mid(y \mid 1) \in F$ or $y \mid(x \mid 1) \in F$ by Lemma 12 . Thus, $F$ is a prime filter of $A$ from Lemma 10 (ii).

\section{Fuzzy Filters of Strong Sheffer Stroke Nmv-Algebras}

In this section, fuzzy filters strong Sheffer stroke NMV-algebras are introduced. 
Definition 8. A fuzzy subset $\alpha$ of $A$ is called a fuzzy filter of $A$ if $(F F 1) \alpha(x) \leq \alpha(1)$,

$(F F 2) \min \{\alpha(x), \alpha(x \mid(y \mid 1))\} \leq \alpha(y)$,

for all $x, y \in A$.

Example 2. Consider the strong Shefeer stroke $N M V$-algebra $A$ in Example 1 . Then a fuzzy subset $\alpha$ of $A$ defined by

$$
\alpha(x)= \begin{cases}0.19, & \text { if } x \approx 0, a, b, d \\ 0.81, & \text { otherwise }\end{cases}
$$

is a fuzzy filter of $A$.

Lemma 13. Let $\alpha$ be a fuzzy filter of $A$. Then

(1) if $x \leq y$, then $\alpha(x) \leq \alpha(y)$,

(2) $\alpha(x \mid(y \mid 1))=\alpha(1)$ implies $\alpha(x) \leq \alpha(y)$,

(3) $\alpha((x \mid y) \mid 1)=\alpha(x) \wedge \alpha(y)$,

(4) $\alpha(x \wedge y)=\alpha(x) \wedge \alpha(y)$,

(5) $\alpha(x) \wedge \alpha(x \mid 1)=\alpha(0)$,

(6) $\alpha(x \mid(y \mid 1)) \wedge \alpha(y \mid(z \mid 1)) \leq \alpha(x \mid(z \mid 1))$,

(7) $\alpha(x) \wedge \alpha(x \mid(y \mid 1))=\alpha(y) \wedge \alpha(y \mid(x \mid 1))=\alpha(x) \wedge \alpha(y)$ and

(8) $\alpha((((x \mid 1) \mid y) \mid y) \mid 1)=\alpha(((y \mid 1) \mid x) \mid x) \mid 1)=\alpha(x \wedge y)$,

for all $x, y, z \in A$.

Proof. (1) Let $x \leq y$. Then $x \mid(y \mid 1) \approx 1$ from Lemma 1 Thus,

$$
\begin{aligned}
\alpha(x) & =\min \{\alpha(x), \alpha(1)\} \\
& =\min \{\alpha(x), \alpha(x \mid(y \mid 1))\} \\
& \leq \alpha(y)
\end{aligned}
$$

from (FF1) and (FF2).

(2) Let $\alpha(x \mid(y \mid 1))=\alpha(1)$. Then

$$
\begin{aligned}
\alpha(x) & =\min \{\alpha(x), \alpha(1)\} \\
& =\min \{\alpha(x), \alpha(x \mid(y \mid 1))\} \\
& \leq \alpha(y)
\end{aligned}
$$

from (FF1) and (FF2).

(3) Since $(x \mid y) \mid 1 \leq x$ and $(x \mid y) \mid 1 \leq y$ from Proposition 2, it follows from (1) that $\alpha((x \mid y) \mid 1) \leq \alpha(x)$ and $\alpha((x \mid y) \mid 1) \leq \alpha(y)$. Thus, $\alpha((x \mid y) \mid 1) \leq$ $\alpha(x) \wedge \alpha(y)$. Also,

$$
\begin{aligned}
\alpha(x) \wedge \alpha(y) & =\min \{\alpha(x), \alpha(y)\} \\
& \leq \min \{\alpha((x \mid y) \mid y), \alpha(y)\} \\
& =\min \{\alpha(y), \alpha(y \mid(((x \mid y) \mid 1) \mid 1))\} \\
& =\alpha((x \mid y) \mid 1)
\end{aligned}
$$


from Lemma 2 (v), (1), (n1), (n3) and (FF2), respectively, Hence,

$$
\alpha((x \mid y) \mid 1)=\alpha(x) \wedge \alpha(y),
$$

for all $x, y \in A$.

(4) Since $x \wedge y \leq x$ and $x \wedge y \leq y$, it is obtained from (1) that $\alpha(x \wedge y) \leq$ $\alpha(x)$ and $\alpha(x \wedge y) \leq \alpha(y)$. So, $\alpha(x \wedge y) \leq \alpha(x) \wedge \alpha(y)$. Moreover, since $(x \mid y) \mid 1 \leq x$ and $(x \mid y) \mid 1 \leq y$ from Proposition 2 , we have $(x \mid y) \mid 1 \leq x \wedge y$. Thus, $\alpha(x) \wedge \alpha(y)=\alpha((x \mid y) \mid 1) \leq \alpha(x \wedge y)$ from (3) and (1), respectively. Therefore, $\alpha(x \wedge y)=\alpha(x) \wedge \alpha(y)$, for all $x, y \in A$.

(5) $\alpha(x) \wedge \alpha(x \mid 1)=\alpha((x \mid(x \mid 1)) \mid 1)=\alpha(1 \mid 1)=\alpha(0)$ from (3) and Lemma 2 (i).

(6)

$$
\begin{aligned}
\alpha(x \mid(y \mid 1)) \wedge \alpha(y \mid(z \mid 1)) & =\min \{\alpha(x \mid(y \mid 1)), \alpha(y \mid(z \mid 1))\} \\
& =\min \{\alpha(x \mid(y \mid 1)), \alpha(x \mid((y \mid(z \mid 1)) \mid 1))\} \\
& =\min \{\alpha(x \mid(y \mid 1)), \alpha((x \mid(y \mid 1)) \mid((x \mid(z \mid 1)) \mid 1))\} \\
& \leq \alpha(x \mid(z \mid 1))
\end{aligned}
$$

(7)

from Lemma 2 (iii), (1), Proposition 1 and (FF2).

$$
\begin{aligned}
\alpha(y) \wedge \alpha(y \mid(x \mid 1)) & =\alpha((y \mid(y \mid(x \mid 1))) \mid 1) \\
& =\alpha((((x \mid 1) \mid y) \mid y) \mid 1) \\
& =\alpha(x \wedge y) \\
& =\alpha(x) \wedge \alpha(y),
\end{aligned}
$$

and similarly, $\alpha(x) \wedge \alpha(x \mid(y \mid 1))=\alpha(y) \wedge \alpha(x)=\alpha(x) \wedge \alpha(y)$ from (3), (n1), Lemma 5 and (4), respectively. Thus, $\alpha(x) \wedge \alpha(x \mid(y \mid 1))=\alpha(y) \wedge$ $\alpha(y \mid(x \mid 1))=\alpha(x) \wedge \alpha(y)$, for all $x, y \in A$.

(8) It is proved Lemma 5

Theorem 3. Let $\alpha$ be a fuzzy subset of $A$. Then $\alpha$ is a fuzzy filter of $A$ if and only if

(i) $\alpha$ is order-preserving,

(ii) $\alpha(x) \wedge \alpha(y) \leq \alpha((x \mid y) \mid 1)$, for all $x, y \in A$.

Proof. Let $\alpha$ be a fuzzy filter of $A$. Then it follows from Lemma 13 (1) and (3).

Conversely, let $\alpha$ be a fuzzy subset of $A$ satisfying (i) and (ii). Since $x \leq 1$, it is obtained from (i) that $\alpha(x) \leq \alpha(1)$, for all $x \in A$.

$$
\begin{aligned}
\min \{\alpha(x), \alpha(x \mid(y \mid 1))\} & =\alpha(x) \wedge \alpha(x \mid(y \mid 1)) \\
& \leq \alpha((x \mid(x \mid(y \mid 1))) \mid 1) \\
& =\alpha(y \wedge x) \\
& \leq \alpha(y)
\end{aligned}
$$


from (ii), (n1), Lemma 5 and (i), respectively. Thus, $\alpha$ is a fuzzy filter of $A$.

Theorem 4. Let $\alpha$ be a fuzzy subset of $A$. Then $\alpha$ is a fuzzy filter of $A$ if and only if $x \leq y \mid(z \mid 1)$ implies $\alpha(x) \wedge \alpha(y) \leq \alpha(z)$, for all $x, y, z \in A$.

Proof. Let $\alpha$ be a fuzzy filter of $A$ and $x \leq y \mid(z \mid 1)$. Then $x \mid((y \mid(z \mid 1)) \mid 1) \approx 1$ from Lemma 1 . Since

$$
\begin{aligned}
((x \mid y) \mid 1) \mid(z \mid 1) & \approx((x \mid y) \mid(x \mid y)) \mid(z \mid 1) \\
& \approx x \mid((y \mid(z \mid 1)) \mid(y \mid(z \mid 1))) \\
& \approx x \mid((y \mid(z \mid 1)) \mid 1) \\
& \approx 1
\end{aligned}
$$

from Lemma 2 (viii) and (S3), it follows from Lemma 1 that $(x \mid y) \mid 1 \leq z$. So, $\alpha(x) \wedge \alpha(y)=\alpha((x \mid y) \mid 1) \leq \alpha(z)$ from Lemma 13 (3) and (1), respectively.

Conversely, let $\alpha$ be a fuzzy subset of $A$ such that $x \leq y \mid(z \mid 1)$ implies $\alpha(x) \wedge$ $\alpha(y) \leq \alpha(z)$, for all $x, y, z \in A$. Since $x \leq 1 \approx x|0 \approx x|(1 \mid 1)$, from (n2), it is obtained that $\alpha(x)=\alpha(x) \wedge \alpha(x) \leq \alpha(1)$, for all $x \in A$. Since $x \leq x \vee$ $y \approx(x \mid(y \mid 1)) \mid(y \mid 1)$ from Lemma 5 , it follows that $\min \{\alpha(x), \alpha(x \mid(y \mid 1))\}=\alpha(x) \wedge$ $\alpha(x \mid(y \mid 1)) \leq \alpha(y)$, for all $x, y \in A$. Hence, $\alpha$ is a fuzzy filter of $A$.

Theorem 5. Let $A$ be a strong Sheffer stroke $N M V$-algebra. Then $\alpha$ is a fuzzy filter of $A$ if and only if $\alpha_{a}=\{x \in A: a \leq \alpha(x)\}$ is empty or a filter of $A$, for all $a \in[0,1]$.

Proof. Let $\alpha$ be a fuzzy filter of $A$ and $\alpha_{a}=\{x \in A: a \leq \alpha(x)\} \neq \emptyset$. Suppose that $x \in \alpha_{a}$. Since $a \leq \alpha(x) \leq \alpha(1)$, we have $1 \in \alpha_{a}$. Let $x, x \mid(y \mid 1) \in \alpha_{a}$. So, $a \leq \alpha(x)$ and $a \leq \alpha(x \mid(y \mid 1))$. Since $a \leq \min \{\alpha(x), \alpha(x \mid(y \mid 1))\} \leq \alpha(y)$, it is obtained that $y \in \alpha_{a}$. Hence, $\alpha_{a}$ is a filter of $A$.

Conversely, let $\alpha_{a} \neq \emptyset$ be a filter of $A$. Assume that $x \in \alpha_{a}$ such that $\alpha(1)<$ $\alpha(x)$. If $a=1 / 2(\alpha(1)+\alpha(x))$, then $\alpha(1)<a<\alpha(x)$. Thus, $1 \notin \alpha_{a}$ which is a contradiction with $\left(S_{f}-1\right)$. Hence, $\alpha(x) \leq \alpha(1)$, for all $x \in A$. Suppose that $x, x \mid(y \mid 1) \in \alpha_{a}$ such that $\alpha(y)<\min \{\alpha(x), \alpha(x \mid(y \mid 1))\}$. If $a=1 / 2(\alpha(y)+$ $\min \{\alpha(x), \alpha(x \mid(y \mid 1))\})$, then $\alpha(y)<a<\min \{\alpha(x), \alpha(x \mid(y \mid 1))\} \leq \alpha(x)$ and $\alpha(y)<$ $a<\min \{\alpha(x), \alpha(x \mid(y \mid 1))\} \leq \alpha(x \mid(y \mid 1))$. Thus, $y \notin \alpha_{a}$ which is a contradiction with $\left(S_{f}-2\right)$. So, $\min \{\alpha(x), \alpha(x \mid(y \mid 1))\} \leq \alpha(y)$, for all $x, y \in A$. Therefore, $\alpha$ is a fuzzy filter of $A$.

Lemma 14. Let $\alpha_{a}$ and $\alpha_{b}$ be two filter of $A$ such that $a<b$. Then $\alpha_{a}=\alpha_{b}$ if and only if there exist no $x_{0} \in A$ such that $a \leq \alpha\left(x_{0}\right)<b$.

Proof. Let $\alpha_{a}=\alpha_{b}$ be such that $a<b$. Then $\alpha_{a}=\{x \in A: a \leq \alpha(x)\}=\{x \in A$ : $b \leq \alpha(x)\}=\alpha_{b}$. If there exists $x_{0} \in A$ such that $a \leq \alpha\left(x_{0}\right)<b$, then $x_{0} \notin \alpha_{b}=\alpha_{a}$ which is a contradiction with $x_{0} \in \alpha_{a}$. Thus, there exist no $x_{0} \in A$ such that $a \leq \alpha\left(x_{0}\right)<b$. 
Conversely, suppose that there exist no $x_{0} \in A$ such that $a \leq \alpha\left(x_{0}\right)<b$. Let $\alpha_{a} \neq \alpha_{b}$ be such that $a<b$. Then there exist $x_{0} \in A$ such that $a \leq c=\alpha\left(x_{0}\right)<b$ which is a contradiction. Hence, $\alpha_{a}=\alpha_{b}$.

Corollary 2. Let $\alpha$ be a fuzzy filter of $A$. Then $\alpha_{a}=\alpha_{b}$, for any $a, b \in \operatorname{Im}(\alpha)$ if and only if $a=b$.

Proof. It is obvious that $\alpha_{a}=\alpha_{b}$, for any $a, b \in \operatorname{Im}(\alpha)$ if $a=b$.

Conversely, let $\alpha_{a}=\alpha_{b}$, for any $a, b \in \operatorname{Im}(\alpha)$. Then there exist $x_{0}, x_{1} \in A$ such that $\alpha\left(x_{0}\right)=a$ and $\alpha\left(x_{1}\right)=b$. So, $x_{0} \in \alpha_{a}=\alpha_{b}$ and $x_{1} \in \alpha_{b}=\alpha_{a}$. Thus, $b \leq \alpha\left(x_{0}\right)=a$ and $a \leq \alpha\left(x_{1}\right)=b$ which imply $a=b$.

Lemma 15. Let $\alpha$ be a fuzzy filter of $A$ and $x_{0} \in A$. Then $\alpha\left(x_{0}\right)=a$ if and only if $x_{0} \in \alpha_{a}$ and $x_{0} \notin \alpha_{b}$, for all $a<b$.

Proof. Let $\alpha\left(x_{0}\right)=a$. Since $\alpha\left(x_{0}\right)=a<b$, we get $x_{0} \in \alpha_{a}$ and $x_{0} \notin \alpha_{b}$, for all $a<b$.

Conversely, let $x_{0} \in \alpha_{a}$ and $x_{0} \notin \alpha_{b}$, for all $a<b$. Then $a \leq \alpha\left(x_{0}\right)<b$. If $a \leq \alpha\left(x_{0}\right)=b_{0}$, then $x_{0} \notin \alpha_{b_{0}}$ which is a contradiction. Hence, $\alpha\left(x_{0}\right)=a$.

Let $\alpha$ be a fuzzy subset of $A$. Define a subset

$$
A_{\alpha}=\{x \in A: \alpha(x)=\alpha(1)\}
$$

of $A$.

Lemma 16. Let $F$ be a nonempty subset of $A$ and $\alpha_{F}$ be a fuzzy subset of $A$ by

$$
\alpha_{F}(x)= \begin{cases}a_{1}, & \text { if } x \in F \\ a_{2}, & \text { otherwise }\end{cases}
$$

where $a_{1}, a_{2} \in[0,1]$ such that $a_{1}>a_{2}$. Then $\alpha_{F}$ is a fuzzy filter of $A$ if and only if $F$ is a filter of $A$. Also, $A_{\alpha_{F}}=F$.

Proof. Let $\alpha_{F}$ be a fuzzy filter of $A$. Since $\alpha_{F}(1)=a_{1}$ by (FF1), we get $1 \in$ $F$. Let $x, x \mid(y \mid 1) \in F$. Then $\alpha_{F}(x)=a_{1}$ and $\alpha_{F}(x \mid(y \mid 1))=a_{1}$. Since $a_{1}=$ $\min \left\{\alpha_{F}(x), \alpha_{F}(x \mid(y \mid 1))\right\} \leq \alpha(y)$, we have $\alpha_{F}(y)=a_{1}$, i.e., $y \in F$.

Conversely, let $F$ be a filter of $A$. Since $1 \in F, \alpha_{F}(x) \leq \alpha_{F}(1)=a_{1}$, for all $x \in A$. Let $\min \left\{\alpha_{F}(x), \alpha_{F}(x \mid(y \mid 1))\right\}=a_{1}$. Then $\alpha_{F}(x)=a_{1}=\alpha_{F}(x \mid(y \mid 1))$ which means that $x \in F$ and $x \mid(y \mid 1) \in F$. So, $y \in F$ which implies $\alpha_{F}(y)=a_{1}$. Thus, $\min \left\{\alpha_{F}(x), \alpha_{F}(x \mid(y \mid 1))\right\} \leq \alpha(y)$. Moreover, if $\min \left\{\alpha_{F}(x), \alpha_{F}(x \mid(y \mid 1))\right\}=a_{2}$, then $\min \left\{\alpha_{F}(x), \alpha_{F}(x \mid(y \mid 1))\right\} \leq \alpha(y)$, for all $x, y \in A$. Hence, $\alpha_{F}$ is a fuzzy filter of $A$.

Since $F$ is a filter of $A$,

$$
\begin{aligned}
A_{\alpha_{F}} & =\left\{x \in A: \alpha_{F}(x)=\alpha_{F}(1)\right\} \\
& =\left\{x \in A: \alpha_{F}(x)=a_{1}\right\} \\
& =\{x \in A: x \in F\} \\
& =A \cap F=F .
\end{aligned}
$$


Definition 9. Let $\alpha$ be a fuzzy filter of $A$. Then $\alpha$ is called a prime fuzzy filter of $A$ if $\alpha(x \vee y)=\alpha(x) \vee \alpha(y)$, for all $x, y \in A$.

Example 3. Consider the strong Sheffer stroke NMV-algebra A in Example 1. Then a fuzzy subset $\alpha_{1}$ of $A$ defined by

$$
\alpha_{1}(x)= \begin{cases}0.007, & \text { if } x \approx 0, a, c, e \\ 0.993, & \text { otherwise }\end{cases}
$$

is a prime fuzzy filter of $A$.

However, a fuzzy subset $\alpha_{2}$ of $A$ defined by

$$
\alpha_{2}(x)= \begin{cases}0.92, & \text { if } x \approx 1 \\ 0.9, & \text { otherwise }\end{cases}
$$

is not a prime fuzzy filter of $A$ since $\alpha_{2}(b \vee e)=\alpha_{2}((b \mid(e \mid 1)) \mid(e \mid 1))=\alpha_{2}(b \mid(b \mid b))=$ $\alpha_{2}(b \mid e)=\alpha_{2}(1) \neq \alpha_{2}(b)=\alpha_{2}(b) \vee \alpha_{2}(e)$.

Theorem 6. Let $\alpha$ be a fuzzy filter of $A$. Then $\alpha$ is a prime fuzzy filter of $A$ if and only if $\alpha(x)=\alpha(1)$ or $\alpha(x \mid 1)=\alpha(1)$, for all $x \in A$.

Proof. Let $\alpha$ be a prime fuzzy filter of $A$. Since

$$
\begin{aligned}
\alpha(x) \vee \alpha(x \mid 1) & =\alpha(x \vee(x \mid 1)) \\
& =\alpha((x \mid((x \mid 1) \mid 1)) \mid((x \mid 1) \mid 1)) \\
& =\alpha(x \mid(x \mid x)) \\
& =\alpha(1)
\end{aligned}
$$

from Lemma 5, (n1), (n3) and Lemma2 (ix), it follows that $\alpha(x)=\alpha(1)$ or $\alpha(x \mid 1)=$ $\alpha(1)$, for all $x \in A$.

Conversely, let $\alpha$ be a fuzzy filter of $A$ such that $\alpha(x)=\alpha(1)$ or $\alpha(x \mid 1)=\alpha(1)$, for all $x \in A$. Since $x \leq x \vee y$ and $y \leq x \vee y$, it follows from Lemma 13 (1) that $\alpha(x) \leq \alpha(x \vee y)$ and $\alpha(y) \leq \alpha(x \vee y)$, and so, $\alpha(x) \vee \alpha(y) \leq \alpha(x \vee y)$, for all $x, y \in A$. If $\alpha(x)=\alpha(1)$ or $\alpha(y)=\alpha(1)$, then $\alpha(x \vee y) \leq \alpha(x) \vee \alpha(y)$ from (FF1). If $\alpha(x) \neq \alpha(1)$ and $\alpha(y) \neq \alpha(1)$, then $\alpha(x \mid 1)=\alpha(1)$ and $\alpha(y \mid 1)=\alpha(1)$. Since

$$
\begin{aligned}
\alpha(x \vee y) & =\alpha(y \vee x) \\
& =\alpha(1) \wedge \alpha(y \vee x) \\
& =\alpha(x \mid 1) \wedge \alpha(y \vee x) \\
& =\alpha(((x \mid 1) \mid(y \vee x)) \mid 1) \\
& =\alpha(((x \mid 1) \mid((y \mid(x \mid 1)) \mid(x \mid 1))) \mid 1) \\
& =\alpha((y \mid(x \mid 1)) \mid 1) \\
& \leq \alpha(y),
\end{aligned}
$$

and similarly, $\alpha(x \vee y) \leq \alpha(x)$ from Lemma 13 (1) and (3), Lemma 5. (n1), (n3), Lemma 2 (iv), (vii) and (ix), it is obtained that $\alpha(x \vee y) \leq \alpha(x) \vee \alpha(y)$. Hence, $\alpha(x \vee y)=\alpha(x) \vee \alpha(y)$, for all $x, y \in A$, i.e., $F$ is a prime fuzzy filter of $A$. 
Theorem 7. Let $\alpha$ be a fuzzy filter of $A$. Then $\alpha$ is a prime fuzzy filter of $A$ if and only if $\alpha(x) \neq \alpha(1)$ and $\alpha(y) \neq \alpha(1)$ imply $\alpha(x \mid(y \mid 1))=\alpha(1)$ and $\alpha(y \mid(x \mid 1))=\alpha(1)$, for all $x, y \in A$.

Proof. Let $\alpha$ be a prime fuzzy filter of $A$ and $\alpha(x) \neq \alpha(1)$ and $\alpha(y) \neq \alpha(1)$. Then $\alpha(x \mid 1)=\alpha(1)$ and $\alpha(y \mid 1)=\alpha(1)$ from Theorem 6. Since $(x \mid 1) \mid((x \mid(y \mid 1)) \mid 1) \approx 1$ and $(y \mid 1) \mid((y \mid(x \mid 1)) \mid 1) \approx 1$ from (n5), it follows from (FF2) that

$$
\alpha(1)=\min \{\alpha(1), \alpha(1)\}=\min \{\alpha(x \mid 1), \alpha((x \mid 1) \mid((x \mid(y \mid 1)) \mid 1))\} \leq \alpha(x \mid(y \mid 1))
$$

and

$$
\alpha(1)=\min \{\alpha(1), \alpha(1)\}=\min \{\alpha(y \mid 1), \alpha((y \mid 1) \mid((y \mid(x \mid 1)) \mid 1))\} \leq \alpha(y \mid(x \mid 1)),
$$

respectively. Thus, $\alpha(x \mid(y \mid 1))=\alpha(1)$ and $\alpha(y \mid(x \mid 1))=\alpha(1)$ from (FF1).

Conversely, let $\alpha$ be a fuzzy filter of $A$ such that $\alpha(x) \neq \alpha(1)$ and $\alpha(y) \neq$ $\alpha(1)$ imply $\alpha(x \mid(y \mid 1))=\alpha(1)$ and $\alpha(y \mid(x \mid 1))=\alpha(1)$, for all $x, y \in A$. If $\alpha(x) \neq$ $\alpha(1)$ and $\alpha(1 \mid 1)=\alpha(0) \neq \alpha(1)$ for any $x \in A$, then $\alpha(x \mid 1)=\alpha(x \mid(0 \mid 1))=\alpha(1)$ and $\alpha(0 \mid(x \mid 1))=\alpha(1)$ from (n1) and (n2). Also, if $\alpha(x \mid 1) \neq \alpha(1)$ and $\alpha(1 \mid 1)=$ $\alpha(0) \neq \alpha(1)$ for any $x \in A$, then $\alpha(x)=\alpha((x \mid 1) \mid 1)=\alpha((x \mid 1) \mid(0 \mid 1))=\alpha(1)$ and $\alpha(0 \mid((x \mid 1) \mid 1))=\alpha(1)$ from (n1)-(n3). Therefore, $\alpha(x)=\alpha(1)$ or $\alpha(x \mid 1)=\alpha(1)$, for all $x \in A$. Hence, $\alpha$ is a prime fuzzy filter of $A$ by Theorem 6 .

Corollary 3. Let $\alpha$ be a fuzzy filter of $A$. Then $\alpha$ is a prime fuzzy filter of $A$ if and only if $\alpha(x \vee(x \mid 1))=\alpha(1)$, for all $x, y \in A$.

Theorem 8. Let $\alpha$ be a fuzzy filter of $A$. Then $\alpha$ is a prime fuzzy filter of $A$ if and only if $\alpha(x \mid(y \mid 1))=\alpha(1)$ or $\alpha(y \mid(x \mid 1))=\alpha(1)$, for all $x, y \in A$.

Proof. Let $\alpha$ be a prime fuzzy filter of $A$. Since

$$
\begin{aligned}
\alpha(x \mid(y \mid 1)) \vee \alpha(y \mid(x \mid 1))= & \alpha((x \mid(y \mid 1)) \vee(y \mid(x \mid 1))) \\
= & \alpha(((x \mid(y \mid 1)) \mid((y \mid(x \mid 1)) \mid 1)) \mid((y \mid(x \mid 1)) \mid 1)) \\
= & \alpha(((x \mid(y \mid y)) \mid((y \mid(x \mid x)) \mid(y \mid(x \mid x)))) \mid((y \mid(x \mid x)) \mid(y \mid(x \mid x)))) \\
= & \alpha(((((x \mid(y \mid y)) \mid(x \mid x)) \mid((x \mid(y \mid y)) \mid \\
& (x \mid x))) \mid y) \mid((y \mid(x \mid x)) \mid(y \mid(x \mid x)))) \\
= & \alpha((y \mid(x \mid x)) \mid((y \mid(x \mid x)) \mid(y \mid(x \mid x)))) \\
= & \alpha(1)
\end{aligned}
$$

from Lemma 5 . Lemma2 (viii), (ix) and (S1)-(S3), it follows that $\alpha(x \mid(y \mid 1))=\alpha(1)$ or $\alpha(y \mid(x \mid 1))=\alpha(1)$, for all $x, y \in A$.

Conversely, let $\alpha$ be a fuzzy filter of $A$ such that $\alpha(x \mid(y \mid 1))=\alpha(1)$ or $\alpha(y \mid(x \mid 1))=$ $\alpha(1)$, for all $x, y \in A$. By substituting $[y:=x \mid 1]$ in the hypothesis, we have $\alpha(1)=$ $\alpha(x \mid((x \mid 1) \mid 1))=\alpha(x \mid x)=\alpha(x \mid 1)$ and $\alpha(1)=\alpha((x \mid 1) \mid(x \mid 1))=\alpha((x \mid x) \mid(x \mid x))=$ $\alpha(x)$ from (n3), Lemma 2 (viii) and (S2). Thus, $\alpha$ is a prime fuzzy filter of $A$. 
Corollary 4. Let $\alpha$ be a fuzzy filter of $A$. Then $\alpha$ is a prime fuzzy filter of $A$ if and only if $\alpha(x \mid(y \mid 1)) \vee \alpha(y \mid(x \mid 1))=\alpha(1)$, for all $x, y \in A$.

Theorem 9. Let $A$ be a strong Sheffer stroke $N M V$-algebra. Then $\alpha$ is a prime fuzzy filter of $A$ if and only if $\alpha_{a}$ is empty or a prime filter of $A$, for all $a \in[0,1]$.

Proof. Let $\alpha$ be a prime fuzzy filter of $A$ and $\alpha_{a} \neq \emptyset$. Assume that $x \vee y \in \alpha_{a}$. Since $a \leq \alpha(x \vee y)=\alpha(x) \vee \alpha(y)$, it follows that $a \leq \alpha(x)$ or $a \leq \alpha(y)$. Thus, $x \in \alpha_{a}$ or $y \in \alpha_{a}$ which imply that $\alpha_{a}$ is a prime filter of $A$.

Conversely, $\alpha_{a} \neq \emptyset$ be a prime filter of $A$ and $a=\alpha(x \vee y)$. Since $x \vee y \in \alpha_{a}$, it is obtained that $x \in \alpha_{a}$ or $y \in \alpha_{a}$. Hence, $a \leq \alpha(x)$ or $a \leq \alpha(y)$, and so, $\alpha(x \vee y)=a \leq \alpha(x) \vee \alpha(y)$. Since $x \leq x \vee y$ and $y \leq x \vee y$, we get from Lemma 13 (1) that $\alpha(x) \leq \alpha(x \vee y)$ and $\alpha(y) \leq \alpha(x \vee y)$. So, $\alpha(x) \vee \alpha(y) \leq \alpha(x \vee y)$. Therefore, $\alpha(x \vee y)=\alpha(x) \vee \alpha(y)$ which means that $\alpha$ is a prime fuzzy filter of $A$.

Corollary 5. Let $A$ be a strong Sheffer stroke $N M V$-algebra. Then $\alpha$ is a (prime) fuzzy filter of $A$ if and only if $\alpha_{\alpha_{(1)}}$ is a (prime) filter of $A$.

Corollary 6. Let $F$ be a nonempty subset of $A$. Then $F$ is a (prime) filter of $A$ if and only if the characteristic function $\chi_{F}$ of $F$ is a (prime) fuzzy filter of $A$.

Corollary 7. Let $F$ be a nonempty subset of $A$ and $\alpha_{F}$ be a fuzzy subset of $A$ by

$$
\alpha_{F}(x)= \begin{cases}a_{1}, & \text { if } x \in F \\ a_{2}, & \text { otherwise }\end{cases}
$$

where $a_{1}, a_{2} \in[0,1]$ such that $a_{1}>a_{2}$. Then $\alpha_{F}$ is a prime fuzzy filter of $A$ if and only if $F$ is a prime filter of $A$.

Proof. Let $\alpha_{F}$ be a prime fuzzy filter of $A$. It is obvious that $F$ is a filter of $A$ by Lemma 16 Since $\alpha_{F}(x)=\alpha_{F}(1)=a_{1}$ or $\alpha_{F}(x \mid 1)=\alpha_{F}(1)=a_{1}$ from $\left(S_{f}-1\right)$, it follows that $x \in F$ or $x \mid 1 \in F$ which means that $F$ is a prime filter of $A$ by Lemma 8.

Let $F$ be a prime filter of $A$. It is clear that $\alpha_{F}$ is a fuzzy filter of $A$ by Lemma 16 Since $x \in F$ or $x \mid 1 \in F$, for all $x \in A$, it is obtained from $\left(S_{f}-1\right)$ that $\alpha_{F}(x)=a_{1}=\alpha_{F}(1)$ or $\alpha_{F}(x \mid 1)=a_{1}=\alpha_{F}(1)$ which means that $\alpha_{F}$ is a prime fuzzy filter of $A$ by Theorem 6 .

Theorem 10. Let $A$ be a strong Sheffer stroke NMV-algebra. Then the following conditions are equivalent:

(1) A is totally ordered.

(2) Every fuzzy filter of $A$ is prime.

(3) $\{1\}$ is a prime filter of $A$.

Proof. Let $A$ be a strong Sheffer stroke NMV-algebra.

$(1) \Rightarrow(2)$ Let $A$ be totally ordered and $\alpha$ be a fuzzy filter of $A$. Then $x \leq y$ or $y \leq x$, for all $x, y \in A$. Since $x \mid(y \mid 1) \approx 1$ or $y \mid(x \mid 1) \approx 1$ from Lemma 1 , it follows 
that $\alpha(x \mid(y \mid 1))=\alpha(1)$ or $\alpha(y \mid(x \mid 1))=\alpha(1)$ for all $x, y \in A$ which means that $\alpha$ is a prime fuzzy filter of $A$ from Theorem 8 .

$(2) \Rightarrow(3)$ Let every fuzzy filter of $A$ be prime. Then $\chi_{\{1\}}$ is a prime fuzzy filter of $A$. Thus, $\{1\}$ is a prime filter of $A$ by Corollary 6 .

(3) $\Rightarrow(1)$ Let the filter $\{1\}$ of $A$ be prime. Then $\chi_{\{1\}}$ is a prime fuzzy filter of $A$ by Corollary 6. Since $\chi_{\{1\}}(x \mid(y \mid 1)) \vee \chi_{\{1\}}(y \mid(x \mid 1))=\chi_{\{1\}}(1)=1$ from Corollary 4. it follows that $\chi_{\{1\}}(x \mid(y \mid 1))=1$ or $\chi_{\{1\}}(y \mid(x \mid 1))=1$, for all $x, y \in A$. Thus, $x \mid(y \mid 1) \approx 1$ or $y \mid(x \mid 1) \approx 1$ which implies that $x \leq y$ or $y \leq x$ from Lemma 1 . Hence, $A$ is totally ordered.

Let $h$ be an endomorphism on $A$ and $\alpha$ be a fuzzy subset of $A$. Define a new fuzzy subset of $A$ by

$$
\alpha_{h}(x)=\alpha(h(x))
$$

for all $x \in A$.

Theorem 11. Let $h$ be a surjective endomorphism on $A$. Then $\alpha$ is a (prime) fuzzy filter of $A$ if and only if $\alpha_{h}$ is a (prime) fuzzy filter of $A$.

Proof. $(\Rightarrow)$ Let $h$ be a surjective endomorphism on $A$ and $\alpha$ be a fuzzy filter of $A$. Then $\alpha_{h}(x)=\alpha(h(x)) \leq \alpha(1)=\alpha(h(1))=\alpha_{h}(1)$, for all $x \in A$. Also,

$$
\begin{aligned}
\min \left\{\alpha_{h}(x), \alpha_{h}(x \mid(y \mid 1))\right\} & =\min \{\alpha(h(x)), \alpha(h(x \mid(y \mid 1)))\} \\
& =\min \{\alpha(h(x)), \alpha(h(x) \mid(h(y) \mid h(1)))\} \\
& \leq \alpha(h(y)) \\
& =\alpha_{h}(y),
\end{aligned}
$$

for all $x, y \in A$. Thus, $\alpha_{h}$ is a fuzzy filter of $A$. If $\alpha$ is prime, then $\alpha_{h}(x)=$ $\alpha(h(x))=\alpha(1)=\alpha(h(1))=\alpha_{h}(1)$ or $\alpha_{h}(x \mid 1)=\alpha(h(x \mid 1))=\alpha(h(x) \mid h(1))=$ $\alpha(h(x) \mid 1)=\alpha(1)=\alpha(h(1))=\alpha_{h}(1)$, for all $x \in A$, for all $x \in A$ so that $\alpha_{h}$ is prime.

$(\Leftarrow)$ Let $h$ be a surjective endomorphism on $A$ and $\alpha_{h}$ be a fuzzy filter of $A$. Then $\alpha(x)=\alpha(h(a))=\alpha_{h}(a) \leq \alpha_{h}(1)=\alpha(h(1))=\alpha(1)$ and

$$
\begin{aligned}
\min \{\alpha(x), \alpha(x \mid(y \mid 1))\} & =\min \{\alpha(h(a)), \alpha(h(a) \mid(h(b) \mid h(1)))\} \\
& =\min \{\alpha(h(a)), \alpha(h(a \mid(b \mid 1)))\} \\
& =\min \left\{\alpha_{h}(a), \alpha_{h}(a \mid(b \mid 1))\right\} \\
& \leq \alpha_{h}(b) \\
& =\alpha(h(b)) \\
& =\alpha(y)
\end{aligned}
$$

where $x=h(a)$ and $y=h(b)$, for all $x, y, a, b \in A$. If $\alpha_{h}$ is prime, then $\alpha(x)=$ $\alpha(h(a))=\alpha_{h}(a)=\alpha_{h}(1)=\alpha(h(1))=\alpha(1)$ or $\alpha(x \mid 1)=\alpha(h(a) \mid h(1))=\alpha(h(a \mid 1))=$ $\alpha_{h}(a \mid 1)=\alpha_{h}(1)=\alpha(h(1))=\alpha(1)$, for all $x, a \in A$, for all $x \in A$. Hence, $\alpha$ is prime. 
Theorem 12. Let $h$ be an automorphism on $A$ and $\alpha$ be a fuzzy filter of $A$. Then $\alpha_{h}=\alpha$ if and only if $h\left(\alpha_{a}\right)=\alpha_{a}$, for any $a \in \operatorname{Im}(\alpha)$.

Proof. Let $\alpha_{h}=\alpha, a \in \operatorname{Im}(\alpha)$ and $x \in \alpha_{a}$. Then $h(x) \in h\left(\alpha_{a}\right)$. Since $a \leq \alpha(x)=$ $\alpha_{h}(x)=\alpha(h(x))$, it follows that $h(x) \in \alpha_{a}$, i.e., $h\left(\alpha_{a}\right) \subseteq \alpha_{a}$. Let $x \in \alpha_{a}$ and $y \in A$ such that $h(y)=x$. Since $a \leq \alpha(x)=\alpha(h(y))=\alpha_{h}(y)=\alpha(y)$, it is obtained that $y \in \alpha_{a}$. Then $x=h(y) \in h\left(\alpha_{a}\right)$ which implies that $\alpha_{a} \subseteq h\left(\alpha_{a}\right)$. Thus, $h\left(\alpha_{a}\right)=\alpha_{a}$, for any $a \in \operatorname{Im}(\alpha)$.

Conversely, let $h\left(\alpha_{a}\right)=\alpha_{a}$, for any $a \in \operatorname{Im}(\alpha)$ and $\alpha(x)=a$. By Lemma 15 $x \in \alpha_{a}$ and $x \notin \alpha_{b}$, for all $a \leq b$. Since $h(x) \in h\left(\alpha_{a}\right)=\alpha_{a}$, we have $a \leq$ $\alpha(h(x))=\alpha_{h}(x)$. Suppose that $\alpha_{h}(x)=b$. Then $\alpha(h(x))=\alpha_{h}(x)=b$, and so, $h(x) \in \alpha_{b}=h\left(\alpha_{b}\right)$. Since $h$ is an automorphism, we get $x \in \alpha_{b}$ which is a contradiction. Thus, $\alpha_{h}(x)=\alpha(h(x))=a=\alpha(x)$, for all $x \in A$, i.e., $\alpha_{h}=\alpha$.

Definition 10. Let $\alpha$ be a fuzzy filter of $A$. Define the binary relation $\sim_{\alpha}$ on $A$ by for all $x, y \in A$

$$
x \sim_{\alpha} y \text { if and only if } \alpha(x \mid(y \mid 1))=\alpha(1)=\alpha(y \mid(x \mid 1)) .
$$

Example 4. Consider the strong Sheffer stroke $N M V$-algebra $A$ in Example 1 . For a fuzzy filter $\alpha$ of $A$ by

$$
\alpha(x)= \begin{cases}0.87, & \text { if } x \approx d, 1 \\ 0.03, & \text { otherwise },\end{cases}
$$

$\sim_{\alpha}=\{(0,0),(a, a),(b, b),(c, c),(d, d),(e, e),(f, f),(1,1),(d, 1),(1, d),(c, 0),(0, c),(a$, $e),(e, a),(b, f),(f, b)\}$ is a binary relation on $A$.

Lemma 17. Let $\alpha$ be a fuzzy filter of $A$ and the binary relation $\sim_{\alpha}$ be defined as (2). Then $\sim_{\alpha}$ is a congruence relation on $A$.

Proof. - Reflexive: Since $\alpha(x \mid(x \mid 1))=\alpha(1)$ from Lemma 2 (i), it follows that $x \sim_{\alpha} x$, for all $x \in A$.

- Let $x \sim_{\alpha} y$. Then $\alpha(x \mid(y \mid 1))=\alpha(1)=\alpha(y \mid(x \mid 1))$. Since $\alpha(y \mid(x \mid 1))=\alpha(1)=$ $\alpha(x \mid(y \mid 1))$, we get $y \sim_{\alpha} x$.

- Let $x \sim_{\alpha} y$ and $y \sim_{\alpha} z$. Then $\alpha(x \mid(y \mid 1))=\alpha(1)=\alpha(y \mid(x \mid 1))$ and $\alpha(y \mid(z \mid 1))=$ $\alpha(1)=\alpha(z \mid(y \mid 1))$. Since $\alpha(1)=\alpha(1) \wedge(1)=\alpha(x \mid(y \mid 1)) \wedge \alpha(y \mid(z \mid 1)) \leq \alpha(x \mid(z \mid 1))$ and $\alpha(1)=\alpha(1) \wedge(1)=\alpha(z \mid(y \mid 1)) \wedge \alpha(y \mid(x \mid 1)) \leq \alpha(z \mid(x \mid 1))$ from Lemma 13 (6), it is obtained that $\alpha(x \mid(z \mid 1))=\alpha(1)=\alpha(z \mid(x \mid 1))$. Thus, $x \sim_{\alpha} z$.

Hence, $\sim_{\alpha}$ is an equivalence relation on $A$.

Let $x \sim_{\alpha} y$ and $z \sim_{\alpha} t$. Then $\alpha(x \mid(y \mid 1))=\alpha(1)=\alpha(y \mid(x \mid 1))$ and $\alpha(z \mid(t \mid 1))=$ $\alpha(1)=\alpha(t \mid(z \mid 1))$.

(a) It follows from (n1), (n3) and Lemma 2 (xiii) that $x|(y \mid 1) \approx(y \mid 1)|((x \mid 1) \mid 1) \leq$ $(z \mid((y \mid 1) \mid 1))|((z \mid((x \mid 1) \mid 1)) \mid 1) \approx(y \mid z)|((x \mid z) \mid 1)$, and similarly, $y \mid(x \mid 1) \leq(x \mid z)$ $\mid((y \mid z) \mid 1)$. Since $\alpha((x \mid z) \mid((y \mid z) \mid 1))=\alpha(1)=\alpha((y \mid z) \mid((x \mid z) \mid 1))$ from Lemma 13 (1) and (FF1), it is obtained $x\left|z \sim_{\alpha} y\right| z$. 
(b) By substituting $[x:=z],[y:=t]$ and $[z:=y]$ in (a), simultaneously, it follows from $(\mathrm{n} 1)$ that $y\left|z \sim_{\alpha} y\right| t$.

Therefore, $x\left|z \sim_{\alpha} y\right| t$ from the transitivity of $\sim_{\alpha}$, and so, $\sim_{\alpha}$ is a congruence relation on $A$.

Theorem 13. Let $\alpha$ be a fuzzy filter of $A$ and $\sim$ be a congruence relation on $A$ defined by $\alpha$. Then $\left(A / \sim,\left.\right|_{\sim},[1]_{\sim}\right)$ is also a strong Sheffer stroke $N M V$-algebra where $A / \sim=\left\{[x]_{\sim}: x \in A\right\}$, the strong Sheffer stroke $\left.\right|_{\sim}$ on $A / \sim$ is defined by $[x]_{\sim} \mid \sim[y]_{\sim}=[x \mid y]_{\sim}$, for all $x, y \in A$. Also, a relation $\preceq$ defined by $[x]_{\sim} \preceq[y]_{\sim} \Leftrightarrow$ $\alpha(x \mid(y \mid 1))=\alpha(1)$, for all $x, y \in A$, is a partial order on $A / \sim$ and $[1]_{\sim}$ is the greatest element and $[0]_{\sim}$ is the least element of $A / \sim$.

Proof. Let $\alpha$ be a fuzzy filter of $A, \sim$ be a congruence relation on $A$ defined by $\alpha$ and the binary operation $\left.\right|_{\sim}$ be defined by $\left.[x]_{\sim}\right|_{\sim}[y]_{\sim}=[x \mid y]_{\sim}$, for all $x, y \in A$. Since

$(\mathrm{n} 1)($ and $(\mathrm{S} 1)):\left.[x]_{\sim}\right|_{\sim}[y]_{\sim}=[x \mid y]_{\sim}=[y \mid x]_{\sim}=\left.[y]_{\sim}\right|_{\sim}[x]_{\sim}$,

(n2): $\left.[x]_{\sim}\right|_{\sim}[0]_{\sim}=[x \mid 0]_{\sim}=[1]_{\sim}$,

(n3): $\left.\left(\left.[x]_{\sim}\right|_{\sim}[1]_{\sim}\right)\right|_{\sim}[1]_{\sim}=[(x \mid 1) \mid 1]_{\sim}=[x]_{\sim}$,

(n4):

$$
\begin{aligned}
\left.\left(\left.\left(\left.[x]_{\sim}\right|_{\sim}[1]_{\sim}\right)\right|_{\sim}[y]_{\sim}\right)\right|_{\sim}[y]_{\sim} & =\left[\left.((x \mid 1) \mid y)\right|_{\sim}\right]_{\sim} \\
& =[((y \mid 1) \mid x) \mid x]_{\sim} \\
& =\left.\left(\left.\left([y]_{\sim} \mid \sim[1]_{\sim}\right)\right|_{\sim}[x]_{\sim}\right)\right|_{\sim}[x]_{\sim},
\end{aligned}
$$

(n5): $\left.\left(\left.[x]_{\sim}\right|_{\sim}[1]_{\sim}\right)\right|_{\sim}\left(\left.\left(\left.[x]_{\sim}\right|_{\sim}[y]_{\sim}\right)\right|_{\sim}[1]_{\sim}\right)=[(x \mid 1) \mid((x \mid y) \mid 1)]_{\sim}=[1]_{\sim}$

(n6):

$$
\begin{aligned}
& {\left.[x]_{\sim}\right|_{\sim}\left(\left.\left(\left.\left(\left.\left(\left.\left.\left(\left.[x]_{\sim}\right|_{\sim}[y]_{\sim}\right)\right|_{\sim}\right|_{\sim}[y]_{\sim}\right)\right|_{\sim}[z]_{\sim}\right)\right|_{\sim}[z]_{\sim}\right)\right|_{\sim}[1]_{\sim}\right)} \\
& =[x \mid(((((x \mid y) \mid y) \mid z) \mid z) \mid 1)]_{\sim} \\
& =[1]_{\sim},
\end{aligned}
$$

$(\mathrm{S} 2):\left.\left(\left.[x]_{\sim}\right|_{\sim}[x]_{\sim}\right)\right|_{\sim}\left(\left.[x]_{\sim}\right|_{\sim}[y]_{\sim}\right)=[(x \mid x) \mid(x \mid y)]_{\sim}=[x]_{\sim}$,

$$
\begin{aligned}
{\left.[x]_{\sim}\right|_{\sim}\left(\left.\left([y]_{\sim} \mid \sim[z]_{\sim}\right)\right|_{\sim}\left([y]_{\sim} \mid \sim[z]_{\sim}\right)\right) } & =[x \mid((y \mid z) \mid(y \mid z))]_{\sim} \\
& =\left[\left.((x \mid y) \mid(x \mid y))\right|_{\sim}\right. \\
& =\left.\left(\left.\left(\left.[x]_{\sim}\right|_{\sim}[y]_{\sim}\right)\right|_{\sim}\left(\left.[x]_{\sim}\right|_{\sim}[y]_{\sim}\right)\right)\right|_{\sim}[z]_{\sim}
\end{aligned}
$$

and

(S4):

$\left.\left(\left.[x]_{\sim}\right|_{\sim}\left(\left.\left(\left.[x]_{\sim}\right|_{\sim}[x]_{\sim}\right)\right|_{\sim}\left(\left.[y]_{\sim}\right|_{\sim}[y]_{\sim}\right)\right)\right)\right|_{\sim}\left(\left.[x]_{\sim}\right|_{\sim}\left(\left.\left(\left.[x]_{\sim}\right|_{\sim}[x]_{\sim}\right)\right|_{\sim}\left(\left.[y]_{\sim}\right|_{\sim}[y]_{\sim}\right)\right)\right)$

$=[(x \mid((x \mid x) \mid(y \mid y))) \mid(x \mid((x \mid x) \mid(y \mid y)))]_{\sim}$

$=[x]_{\sim}$,

for all $x, y, z \in A$, the binary operation $\mid \sim$ is a strong Sheffer stroke.

- Reflexive: $[x]_{\sim} \preceq[x]_{\sim}$ since $\alpha(x \mid(x \mid 1))=\alpha(1)$, from Lemma 2 (i).

- Antisymmetric: let $[x]_{\sim} \preceq[y]_{\sim}$ and $[y]_{\sim} \preceq[x]_{\sim}$. Since $\alpha(x \mid(y \mid 1))=\alpha(1)=$ $\alpha(y \mid(x \mid 1))$, we have $x \sim y$ which implies $[x]_{\sim}=[y]_{\sim}$. 
- Transitive: let $[x]_{\sim} \preceq[y]_{\sim}$ and $[y]_{\sim} \preceq[z]_{\sim}$. Then $\alpha(x \mid(y \mid 1))=\alpha(1)$ and $\alpha(y \mid(z \mid 1))=\alpha(1)$. Since $\alpha(1)=\alpha(1) \wedge \alpha(1)=\alpha(x \mid(y \mid 1)) \wedge \alpha(y \mid(z \mid 1)) \leq \alpha(x \mid(z \mid 1))$ from Lemma 13 (6), it follows from (FF1) that $\alpha(x \mid(z \mid 1))=\alpha(1)$, i.e., $[x]_{\sim} \preceq[z]_{\sim}$.

Thus, $\preceq$ is a partial order on $A / \sim$.

Since $\alpha(x \mid(1 \mid 1))=\alpha(x \mid 0)=\alpha(1)$ from $(\mathrm{n} 2)$, it is ontained that $[x]_{\sim} \preceq[1]_{\sim}$, for all $x \in A$. Thus, $[1]_{\sim}$ is the greatest element, and so, $[0]_{\sim}=[1 \mid 1]_{\sim}=\left.[1]_{\sim}\right|_{\sim}[1]_{\sim}$ is the least element of $A / \sim$.

Example 5. Consider the strong Shefeer stroke $N M V$-algebra $A$ in Example 1 . For a fuzzy filter $\alpha$ of $A$ defined by

$$
\alpha(x)= \begin{cases}1, & \text { if } x \approx f, 1 \\ 0.001, & \text { otherwise }\end{cases}
$$

$\sim_{\alpha}=\{(0,0),(a, a),(b, b),(c, c),(d, d),(e, e),(f, f),(1,1),(f, 1),(1, f),(a, 0),(0, a)$, $(c, e),(e, c),(b, d),(d, b)\}$ is a congruence relation on $A$. Then $\left(A / \sim_{\alpha}, \mid \sim_{\alpha},[1]_{\sim}\right)$ is also a strong Sheffer stroke NMV-algebra with the following Cayley table where $A / \sim_{\alpha}=\left\{[0]_{\sim_{\alpha}},[d]_{\sim_{\alpha}},[e]_{\sim_{\alpha}},[1]_{\sim_{\alpha}}\right\}:$

TABle 2. Cayley table of $\left.\right|_{\sim_{\alpha}}$

\begin{tabular}{c|cccc}
||$_{\sim_{\alpha}}$ & {$[0]_{\sim_{\alpha}}$} & {$[d]_{\sim_{\alpha}}$} & {$[e]_{\sim_{\alpha}}$} & {$[1]_{\sim_{\alpha}}$} \\
\hline$[0]_{\sim_{\alpha}}$ & {$[1]_{\sim_{\alpha}}$} & {$[1]_{\sim_{\alpha}}$} & {$[1]_{\sim_{\alpha}}$} & {$[1]_{\sim_{\alpha}}$} \\
{$[d]_{\sim_{\alpha}}$} & {$[1]_{\sim_{\alpha}}$} & {$[e]_{\sim_{\alpha}}$} & {$[1]_{\sim_{\alpha}}$} & {$[e]_{\sim_{\alpha}}$} \\
{$[e]_{\sim_{\alpha}}$} & {$[1]_{\sim_{\alpha}}$} & {$[1]_{\sim_{\alpha}}$} & {$[d]_{\sim_{\alpha}}$} & {$[d]_{\sim_{\alpha}}$} \\
{$[1]_{\sim_{\alpha}}$} & {$[1]_{\sim_{\alpha}}$} & {$[e]_{\sim_{\alpha}}$} & {$[d]_{\sim_{\alpha}}$} & {$[0]_{\sim_{\alpha}}$}
\end{tabular}

Theorem 14. Let $\alpha$ be a fuzzy filter of $A$. Then $\alpha$ is a prime fuzzy filter of $A$ if and only if $A / \sim_{\alpha}$ is totally ordered and $\left|A / \sim_{\alpha}\right| \leq 2$.

Proof. Let $\alpha$ be a prime fuzzy filter of $A$. By Theorem $8, \alpha(x \mid(y \mid 1))=\alpha(1)$ or $\alpha(y \mid(x \mid 1))=\alpha(1)$. Then $[x]_{\sim} \preceq[y]_{\sim}$ or $[y]_{\sim} \preceq[x]_{\sim}$ which means that $A / \sim_{\alpha}$ is totally ordered. Also, let $\left|A / \sim_{\alpha}\right|>2$. Then $[x]_{\sim_{\alpha}} \in A / \sim_{\alpha}$ such that $[0]_{\sim_{\alpha}}<[x]_{\sim_{\alpha}}<[1]_{\sim_{\alpha}}$. Since $\alpha$ is a prime fuzzy filter of $A$, we have $\alpha(x)=\alpha(1)$ or $\alpha(x \mid 1)=\alpha(1)$. Assume that $\alpha(x \mid 1)=\alpha(1)$. Since $\alpha(x \mid(0 \mid 1))=\alpha(x \mid 1)=\alpha(1)$ and $\alpha(0 \mid(x \mid 1))=\alpha(1)$ from (n2), it follows that $[x]_{\sim_{\alpha}}=[0]_{\sim_{\alpha}}$ which is a contradiction. So, $\left|A / \sim_{\alpha}\right| \leq 2$.

Conversely, let $A / \sim_{\alpha}$ be totally ordered. Then $[x]_{\sim} \preceq[y]_{\sim}$ or $[y]_{\sim} \preceq[x]_{\sim}$, for all $x, y \in A$. Since $\alpha(x \mid(y \mid 1))=\alpha(1)$ or $\alpha(y \mid(x \mid 1))=\alpha(1)$, it is obtained from Theorem 8 that $\alpha$ is a prime fuzzy filter of $A$.

Theorem 15. Let $\left(A,\left.\right|_{A}, 1_{A}\right)$ and $\left(B,\left.\right|_{B}, 1_{B}\right)$ be strong Sheffer stroke $N M V$-algebras, $h: A \longrightarrow B$ be an epimorphism and $\alpha$ be a fuzzy filter of $B$. Then $\alpha \circ h$ is a fuzzy filter of $A$ and $A / \sim_{\alpha \circ h} \cong B / \sim_{\alpha}$. 
Proof. Let $\left(A,\left.\right|_{A}, 1_{A}\right)$ and $\left(B,\left.\right|_{B}, 1_{B}\right)$ be strong Sheffer stroke NMV-algebras, $h$ : $A \longrightarrow B$ be an epimorphism and $\alpha$ be a fuzzy filter of $B$. It is first shown that $\alpha \circ h$ is a fuzzy filter of $A$.

- $\alpha \circ h(x)=\alpha(h(x)) \leq \alpha\left(1_{B}\right)=\alpha\left(h\left(1_{A}\right)\right)=\alpha \circ h\left(1_{A}\right)$ and

$$
\begin{aligned}
\min \left\{\alpha \circ h(x), \alpha \circ h\left(\left.x\right|_{A}\left(\left.y\right|_{A} 1_{A}\right)\right)\right\} & =\min \left\{\alpha(h(x)), \alpha\left(h\left(\left.x\right|_{A}\left(\left.y\right|_{A} 1_{A}\right)\right)\right)\right\} \\
& =\min \left\{\alpha(h(x)), \alpha\left(\left.h(x)\right|_{B}\left(\left.h(y)\right|_{B} h\left(1_{A}\right)\right)\right)\right\} \\
& =\min \left\{\alpha(h(x)), \alpha\left(\left.h(x)\right|_{B}\left(\left.h(y)\right|_{B} 1_{B}\right)\right)\right\} \\
& \leq \alpha(h(y)) \\
& =\alpha \circ h(y),
\end{aligned}
$$

for all $x, y \in A$.

$A / \sim_{\alpha \circ h}$ and $B / \sim_{\alpha}$ are strong Sheffer stroke NMV-algebras by Theorem 13 . Let $f: A / \sim_{\alpha \circ h} \longrightarrow B / \sim_{\alpha}$ be defined by $f\left([x]_{\sim_{\alpha \circ h}}\right)=[h(x)]_{\sim_{\alpha}}$, for all $x \in A$.

- $f$ is well-defined and one-to-one: Let $[x]_{\sim_{\alpha \circ h}},[y]_{\sim_{\alpha \circ h}} \in A / \sim_{\alpha \circ h}$. Then

$$
\begin{aligned}
{[x]_{\sim_{\alpha \circ h}}=[y]_{\sim_{\alpha \circ h}} \Leftrightarrow } & x \sim_{\alpha \circ h} y \\
\Leftrightarrow & \alpha \circ h\left(\left.x\right|_{A}\left(\left.y\right|_{A} 1_{A}\right)\right)=\alpha \circ h\left(1_{A}\right)=\alpha \circ h\left(\left.y\right|_{A}\left(\left.x\right|_{A} 1_{A}\right)\right) \\
\Leftrightarrow & \alpha\left(\left.h(x)\right|_{B}\left(\left.h(y)\right|_{b} h\left(1_{A}\right)\right)\right)=\alpha\left(h\left(1_{A}\right)\right) \\
& =\alpha\left(\left.h(y)\right|_{B}\left(\left.h(x)\right|_{b} h\left(1_{A}\right)\right)\right) \\
\Leftrightarrow & \alpha\left(\left.h(x)\right|_{B}\left(\left.h(y)\right|_{b} 1_{B}\right)\right)=\alpha\left(1_{B}\right)=\alpha\left(\left.h(y)\right|_{B}\left(\left.h(x)\right|_{b} 1_{B}\right)\right) \\
\Leftrightarrow & h(x) \sim_{\alpha} h(y) \\
\Leftrightarrow & {[h(x)]_{\sim_{\alpha}}=[h(y)]_{\sim_{\alpha}} } \\
\Leftrightarrow & f\left([x]_{\sim_{\alpha \circ h}}\right)=f\left([y]_{\sim_{\alpha \circ h}}\right) .
\end{aligned}
$$

- $f$ is a homomorphism: Let $[x]_{\sim_{\alpha \circ h}},[y]_{\sim_{\alpha \circ h}} \in A / \sim_{\alpha \circ h}$. Then

$$
\begin{aligned}
f\left([x]_{\sim_{\alpha \circ h}}||_{\sim_{\alpha \circ h}}[y]_{\sim_{\alpha \circ h}}\right) & =f\left(\left[\left.x\right|_{A} y\right]_{\sim_{\alpha \circ h}}\right) \\
& =[h(x \mid A y)]_{\sim_{\alpha}} \\
& =\left[\left.h(x)\right|_{B} h(y)\right]_{\sim_{\alpha}} \\
& =[h(x)]_{\sim_{\alpha}} \mid{ }_{\sim_{\alpha}}[h(y)]_{\sim_{\alpha}} \\
& =\left.f\left([x]_{\sim_{\alpha \circ h}}\right)\right|_{\sim_{\alpha}} f\left([y]_{\sim_{\alpha \circ h}}\right) .
\end{aligned}
$$

- $f$ is onto: Let $[y]_{\sim_{\alpha}} \in B / \sim_{\alpha}$. Since $h$ is an epimorphism, there exists $x \in A$ such that $h(x)=y$. Thus, there exists $[x]_{\sim_{\alpha \circ h}} \in A / \sim_{\alpha \circ h}$ such that $f\left([x]_{\sim_{\alpha \circ h}}\right)=[h(x)]_{\sim_{\alpha}}=[y]_{\sim_{\alpha}}$.

Theorem 16. The class $\mathcal{F}_{A}$ of all fuzzy filters of $A$ forms a complete lattice.

Proof. Since every fuzzy filter of $A$ is a mapping from $A$ to the interval $[0,1]$ and $[0,1]$ is a complete lattice where $a \vee b=\max \{a, b\}$ and $a \wedge b=\min \{a, b\}$, for all $a, b \in[0,1], \mathcal{F}_{A}$ forms a complete lattice. 


\section{CONCLUSiOn}

In present study, basic definitions and notions of a strong Sheffer stroke NMValgebra are given. Then new properties, various filters, fuzzy filters of a strong Sheffer stroke NMV-algebra and the relationships between them are investigated. We prove that a filter of a strong Sheffer stroke NMV-algebra is prime if and only if it is not contained by another filter of this algebraic structure, and examine some features of a prime filter. Also, it is shown that the quotient structure of a strong Sheffer stroke NMV-algebra defined by a prime filter is totally ordered and it has at most 2 elements. Besides, we define a (prime) fuzzy filter of strong Sheffer stroke NMV-algebras and show that $\alpha$ is a (prime) fuzzy filter of a strong Sheffer stroke NMV-algebra if and only if $\alpha_{a}=\{x \in A: a \leq \alpha(x)\}$ is empty or a (prime) filter of $A$, for all $a \in[0,1]$. It is demonstrated that a fuzzy subset $\alpha_{F}$ is a (prime) fuzzy filter of a strong Sheffer stroke NMV-algebra if and only if $F$ is a (prime) filter of the algebra. Thus, the relationships between filters and fuzzy filters of a strong Sheffer stroke NMV-algebra are stated. We prove that a strong Sheffer stroke NMV-algebra is totally ordered if and only if every fuzzy filter is prime if and only if the filter $\{1\}$ is prime. It is shown that a fuzzy subset $\alpha_{h}$ of a strong Sheffer stroke NMV-algebra is a (prime) fuzzy filter defined by means of a (prime) fuzzy filter $\alpha$ and a surjective endomomorphism $h$ on this algebra, and that $\alpha_{h}=\alpha$ if and only if $h\left(\alpha_{a}\right)=\alpha_{a}$ whenever $h$ is an automorphism on this algebra and $a \in \operatorname{Im}(\alpha)$. By describing a congruence relation on a strong Sheffer stroke NMV-algebra by a fuzzy filter, a quotient structure of a strong Sheffer stroke NMV-algebra is built via the congruence relation. Hence, it is shown that the structure forms a strong Sheffer stroke NMV-algebra. Indeed, we prove that the quotient structure defined by a prime fuzzy filter is totally ordered strong Sheffer stroke NMV-algebra and it has at most 2 elements. Moreover, we present that $\alpha \circ h$ is a fuzzy filter of $A$ and the quotient structures defined by the fuzzy filters $\alpha \circ h$ and $\alpha$ are isomorphic when an epimorphism $h$ between strong Sheffer stroke NMV-algebras $A$ and $B$ and a fuzzy filter $\alpha$ of $B$. Finally, it is easy to see that the class of all fuzzy filters of a strong Sheffer stroke NMV-algebra forms a complete lattice.

In the future works, we wish to investigate annihilators ans stabilizers on strong Sheffer stroke NMV-algebras.

Author Contribution Statements The authors have made equally contributions to the study.

Declaration of Competing Interests There is no declaration of competing interest.

Acknowledgement The authors would like to thank the referees for their helpful suggestions and their valuable comments which helped to improve the manuscript. 


\section{REFERENCES}

[1] Abbott, J. C., Implicational algebras, Bulletin Mathématique de la Société des Sciences Mathématiques de la République Socialiste de Roumanie, 11(1) (1967), 3-23. http://www.jstor.org/stable/43679502

[2] Botur, M., Halaš, R., Commutative basic algebras and non-assocative fuzzy logics, Archive for Mathematical Logic, 48 (2009), 243-255. https://doi.org/10.1007/s00153-009-0125-7

[3] Chajda, I., Sheffer operation in ortholattices, Acta Universitatis Palackianae Olomucensis Facultas Rerum Naturalium Mathematica, (44)(1) (2005) 19-23. http://dml.cz/dmlcz/133381

[4] Chajda, I., Halaš, R., Länger, H., Operations and structures derived from non-associative MV-algebras, Soft Computing, 23(12) (2019), 3935-3944. https://doi.org/10.1007/s00500018-3309-4

[5] Chajda, I., Kühr, J., A non-associative generalization of MV-algebras, Mathematica Slovaca, 57 (2007), 301-312. https://doi.org/10.2478/s12175-007-0024-5

[6] Chajda, I., Länger, H., Properties of non-associative MV-algebras, Mathematica Slovaca, 67 (2017), 1095-1104. https://doi.org/10.1515/ms-2017-0035

[7] Esteva, F., Godo, L., Monoidal t-norm based logic: towards a logic for left-continous t-norms, Fuzzy Sets and Systems, 124 (2001), 271-288. https://doi.org/10.1016/S01650114(01)00098-7

[8] Hájek, P., Metamathematics of Fuzzy Logic, Trends in Logic, vol. 4, Kluwer Academic Publishers, 1998.

[9] McCune, W., Veroff, R., Fitelson, B., Harris, K., Feist, A., Wos, L., Short single axioms for Boolean algebra, Journal of Automated Reasoning, 29(1) (2002), 1-16. https://doi.org/10.1023/A:1020542009983

[10] Oner, T., Katican, T., Borumand Saeid, A., Terziler, M., Filters of strong Sheffer stroke non-associative MV-algebras, Analele Stiintifice ale Universitatii Ovidius Constanta, 29(1) (2021), 143-164. https://doi.org/10.2478/auom-2021-0010

[11] Oner, T., Katican, T. Borumand Saeid, A., Relation between Sheffer stroke operation and Hilbert algebras, Categories and General Algebraic Structures with Applications, 14(1) (2021), 245-268. https://doi.org/10.29252/CGASA.14.1.245

[12] Oner, T., Katican, T., Borumand Saeid, A., Fuzzy filters of Sheffer stroke Hilbert algebras, Journal of Intelligent and Fuzzy Systems, 40(1) (2021), 759-772. https://doi.org/10.3233/JIFS-200760

[13] Oner, T., Katican, T., Borumand Saeid, A., Fuzzy filters of Sheffer stroke BL-algebras, Kragujevac Journal of Mathematics, 47(1) (2023), 39-55.

[14] Oner, T., Katican, T., Borumand Saeid, A., On Sheffer stroke UP-algebras, Discussiones Mathematicae General Algebra and Applications, 41 (2021), 381-394 https://doi.org/10.7151/dmgaa.1368

[15] Oner, T., Katican, T., Rezaei, A., Neutrosophic n-structures on strong Sheffer stroke non-associative MV-algebras, Neutrosophic Sets and Systems, 40 (2021), 235-252. https://doi.org/10.5281/zenodo.4549403

[16] Sheffer, H. M., A set of five independent postulates for Boolean algebras, with application to logical constants, Transactions of the American Mathematical Society, 14(4) (1913), 481488. https://doi.org/10.2307/1988701

[17] Wang, G.-J., Non-classical Mathematical Logic and Approximate Reasoning, Science Press, 2000.

[18] Zadeh, L. A., Fuzzy sets, Information and Control, 8 (1965), 338-353. 